\title{
Tubos rellenos de hormigón expansivo. Aplicación al viaducto sobre el barranco de Erques. Programa experimental e instrumentación in situ
}

\section{Tubes Filled with Expansive Concrete. Application to the Viaduct over the Erques Ravine. Experimental Programme and in-situ Instrumentation}

\author{
Jose Pedro Gutiérrez ${ }^{\mathrm{a}}$, Sonia Martínez ${ }^{\mathrm{a}}$, \\ Juan José Sánchez ${ }^{\mathrm{b}}$, Fco. Javier Barroso ${ }^{\mathrm{a}}$, Luis Echevarría ${ }^{\mathrm{a}}$ \\ a Instituto de Ciencias de la Construcción Eduardo Torroja (IETcc, CSIC) \\ ${ }^{b}$ Ferrovial Construcción, S.A.
}

Recibido el 30 de noviembre de 2020; aceptado el 5 de abril de 2021

\begin{abstract}
RESUMEN
El viaducto sobre el barranco de Erques (Tenerife) es un puente formado por dos arcos con tablero intermedio de $110 \mathrm{~m}$ de longitud y una flecha en el centro de $15 \mathrm{~m}$ respecto al tablero. Cada arco está compuesto por dos tubos de acero, de $1020 \mathrm{~mm}$ de diámetro, rellenos de hormigón expansivo. En este artículo se describe el programa experimental que ha servido de base para la ejecución de los arcos del viaducto. También se detalla la instrumentación realizada para registrar las deformaciones longitudinales y circunferenciales de los tubos y las temperaturas de los mismos durante el proceso de hormigonado y posterior endurecimiento, con el objeto de estimar el efecto expansor del hormigón y comprobar si los resultados son acordes a los determinados en la investigación previa. La caracterización del hormigón expansivo para su aplicación en estructuras tubulares requiere realizar ensayos de expansión libre y confinada del hormigón para ajustar la deformación de expansión necesaria. Estos ensayos se complementaron con otros para determinar la carga de deslizamiento del núcleo de hormigón dentro del tubo de acero a temperatura ambiente y con calentamiento uniforme y diferencial, realizados para evaluar la incidencia de los efectos térmicos en la resistencia a esfuerzos rasantes de la interfase tubo-hormigón.
\end{abstract}

PALABRAS CLAVE: Estructura mixta; tubo acero-hormigón; hormigón expansivo; puente arco; instrumentación.

(C) 2021 Asociación Española de Ingeniería Estructural (ACHE). Publicado por Cinter Divulgación Técnica S.L. Todos los derechos reservados.

\section{ABSTRACT}

The viaduct over the Erques ravine (Tenerife) is a through arch bridge formed by two arcs with $110 \mathrm{~m}$ span and $15 \mathrm{~m}$ of rise at the mid span. Each arch is composed of two steel tubes, of $1020 \mathrm{~mm}$ diameter, filled with expansive concrete. This paper describes the previous experimental program that served as the basis for the execution of the arches of the viaduct. The instrumentation carried out to record the longitudinal and circumferential strains and temperatures outside the tubes during the casting process and subsequent hardening is also detailed, in order to estimate the expansive effect of the concrete and check if the results are according to those determined in the previous research.

The characterization of expansive concrete for its application in tubular structures requires performing free and confined concrete expansion tests, in order to adjust the required expansion strain. These tests were complemented with others used to determine the slip load of the concrete core inside the steel tube at room temperature and with uniform and differential heating, carried out to evaluate the incidence of thermal effects on the resistance capacity to interfacial shear stress forces of the interface tube-concrete.

KEYWORDS: Composite structure; steel-concrete tube; expansive concrete; arch bridge; monitoring.

(C) 2021 Asociación Española de Ingeniería Estructural (ACHE). Published by Cinter Divulgación Técnica S.L. All rights reserved.

Persona de contacto / Corresponding author

Correo-e / email: jpgutierrez@ietcc.csic.es (José Pedro Gutiérrez Jiménez). 
1.

\section{INTRODUCCIÓN}

Se conoce como CFT ("concrete-filled tubes") a elementos estructurales formados por perfiles tubulares de acero rellenos de hormigón. Esta combinación presenta ventajas como el hecho de que, para cargas axiales elevadas, el tubo metálico restringe la deformación lateral del hormigón generando sobre él un efecto de confinamiento, al tiempo que el hormigón limita el pandeo del acero hacia el interior del tubo. Además, el tubo exterior puede soportar cargas durante el proceso de construcción y hace las funciones de encofrado, reduciendo tiempos y costes de ejecución [1].

En los últimos años se han llevado a cabo numerosos estudios teóricos y experimentales acerca del comportamiento estructural de los CFT, [2]. Destacan entre otros, los publicados por Schneider [3], O'Shea et al. [4], Hatzigeorgiou [5-6], de Oliveira et al. [7] y Chacón et al. [8]. Varios autores señalan que en la mejora de la capacidad resistente que se obtiene con el sistema mixto resulta relevante el grado de confinamiento alcanzado en el hormigón, [9]. No obstante, para compresiones sustancialmente inferiores a la carga última, como son las cargas de servicio, este confinamiento estaría cuestionado porque el tubo de acero se puede expandir lateralmente más que el núcleo central de hormigón, al tener el acero y el hormigón distintos coeficientes de Poisson. La propia retracción del núcleo de hormigón y una temperatura medioambiental mayor en la camisa de acero puede dar lugar a la desconexión del tubo perdiendo las ventajas de un sistema mixto. Utilizando un hormigón expansivo se puede generar una deformación tal que, teniendo en cuenta todas las solicitaciones y efectos, quede una deformación remanente que permita la generación de una presión de confinamiento y, en consecuencia, una capacidad para resistir tensiones rasantes en la interfase hormigón-tubo de acero, que pueda evitar o disminuir la utilización de conectores. Además, el confinamiento sobre el hormigón incrementa la capacidad portante del mismo.

Un criterio de dimensionamiento consiste en determinar el valor de expansión que debe generarse en el hormigón para que el balance neto de las deformaciones del acero y del hormigón debido a los diferentes fenómenos (deformación de expansión del hormigón, deformaciones por solicitaciones externas, temperatura, retracción y fluencia) sea positivo. Interesa que la presión de confinamiento resultante genere una capacidad frente a tensiones rasantes mayor o igual que las tensiones rasantes de cálculo. De esta forma el sistema se aproxima a una estructura mixta.

La capacidad de resistir tensiones rasantes en la interfase está regida por la presión de confinamiento que se desarrolla y el coeficiente de rozamiento entre el tubo metálico y el hormigón. El producto de estos dos parámetros indica la capacidad del elemento frente al deslizamiento del núcleo dentro del tubo y, por lo tanto, en ausencia de conectores, marca un límite de capacidad portante del sistema mixto para resistir tensiones rasantes. Independientemente de las estimaciones teóricas, es necesario la comprobación de dicha capacidad mediante ensayos de deslizamiento.

En los estudios de expansión debe tenerse en cuenta que, dado un tipo de acero y una resistencia del hormigón, las tensiones en ambos materiales debidas a la deformación de expansión del hormigón tienen el mismo valor si la relación entre el diámetro exterior del elemento y el espesor del tubo (D/t) permanece constante. Este hecho sirve también para elegir en los programas experimentales una relación $\mathrm{D} / \mathrm{t}$ adecuada y similar a la proyectada en la obra real.

En cuanto a la normativa existente, las estructuras mixtas de hormigón y acero a nivel europeo están reguladas por el Eurocódigo 4, UNE-EN 1994-1-1 [10] donde se recogen aspectos relativos al cálculo para los E.L.U. de pilares mixtos y otros elementos mixtos comprimidos, incluidos los tubos circulares rellenos de hormigón. El acero estructural está recogido en el Eurocódigo 3, UNE-EN 1993-1-3 [11] y el hormigón estructural en el Eurocódigo 2, UNE-EN 1992-1-1 [12], así como en la Instrucción de Hormigón Estructural [13]. Como normativa de referencia a nivel internacional puede citarse el código americano AISC (2010) [14]. A nivel nacional se han publicado guías de referencia sobre hormigón expansivo [15].

No obstante, existe una ausencia de criterios claros de dimensionamiento de tubos rellenos con hormigón expansivo y de una reglamentación adaptada a estos sistemas, lo que hace necesario realizar un esfuerzo de investigación experimental para determinar las variables y parámetros fundamentales que rigen su comportamiento. Así, por ejemplo, una vez elegido el tipo y dosis de aditivo expansivo es necesario verificar experimentalmente las deformaciones de expansión y la capacidad frente al deslizamiento para comprobar la rigidez del tubo y los estados límite últimos del elemento.

Con este objetivo, el IETcc y Ferrovial Construcción, S.A. han colaborado en una investigación sobre elementos mixtos, tubos de acero rellenos de hormigón expansivo, con un programa experimental desarrollado en los laboratorios y naves de ensayo del IETcc, que permitiera evaluar los parámetros fundamentales que rigen el comportamiento de estos sistemas, con aplicación directa a la ejecución de los arcos del viaducto del Barranco de Erques.

Este viaducto, ubicado en la nueva carretera Adeje-Santiago del Teide en Tenerife, es un puente formado por dos arcos con tablero intermedio de $110 \mathrm{~m}$ de longitud, $25.7 \mathrm{~m}$ de ancho y una flecha en el centro de $15 \mathrm{~m}$ respecto al tablero, con una relación flecha luz de 1/7.3, figura 1. Los arcos, de directriz parabólica, se encuentran biempotrados en los arranques. Las cimentaciones de arcos y estribos del tablero son directas en el macizo rocoso.

Cada uno de los arcos está compuesto por dos tubos de acero S355, de $1020 \mathrm{~mm}$ de diámetro y $13 \mathrm{~mm}$ de espesor, rellenos de hormigón. La distancia entre los centros de los tubos de cada arco es de $1.5 \mathrm{~m}$, encontrándose unidos en 13 secciones. El tablero es un emparrillado metálico con losa de compresión hormigonada sobre prelosas y está suspendido de los arcos cada $8 \mathrm{~m}$ mediante dos péndolas inclinadas de cable cerrado de $50 \mathrm{~mm}$ de diámetro, figura 2. El viaducto es un hito tecnológico de la ingeniería estructural española, siendo el primer puente en utilizar la tecnología del hormigón expansivo como relleno de los tubos de acero de los arcos portantes del tablero, prescindiendo de conectores interiores.

La estructura del viaducto es evolutiva, cambiando durante el proceso constructivo. Los arcos pasan por varias fases en su ejecución, conformando distintos sistemas estructurales resistentes y soportando diferentes esfuerzos: esfuerzos de izado y cuelgue durante el montaje de los arcos y en vacío en su posición definitiva; esfuerzos durante el llenado de los tubos; esfuerzos como estructura mixta una vez endurecido el hor- 


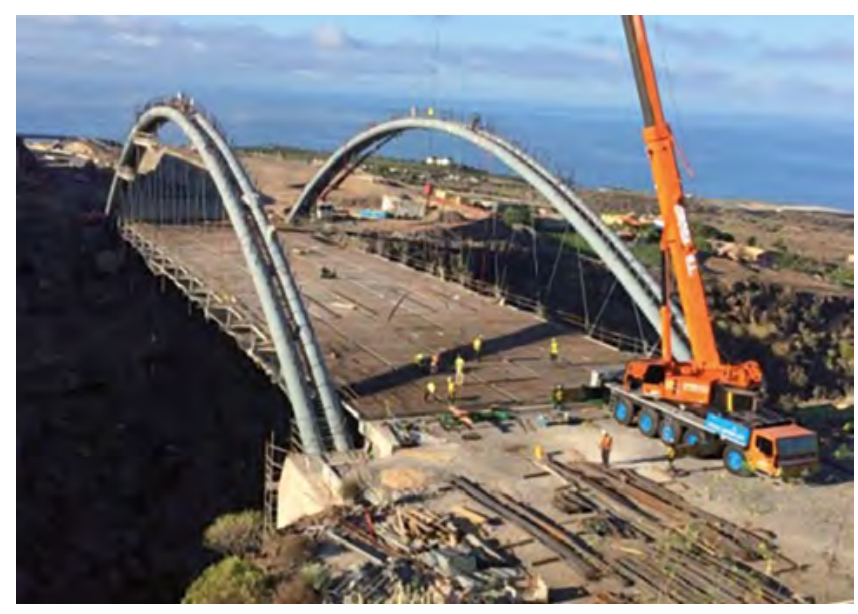

a)

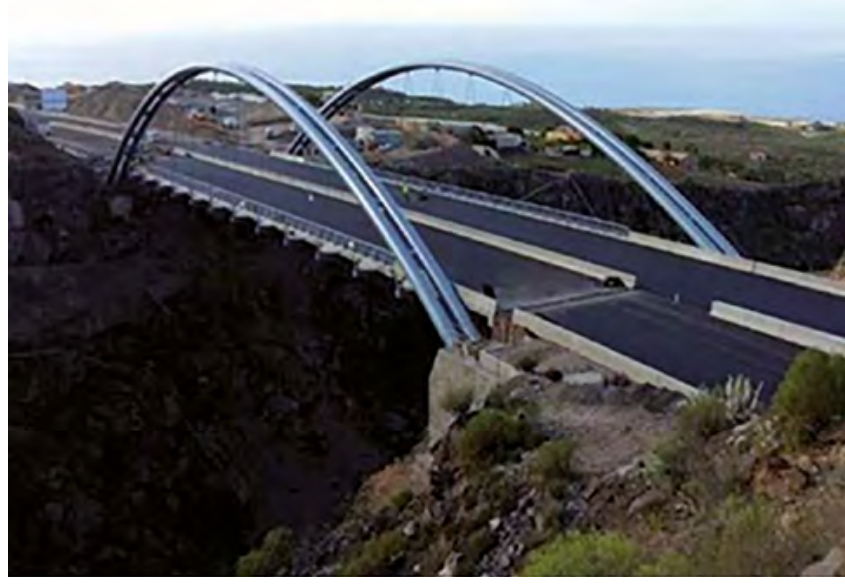

b)

Figura 1. Viaducto del Barranco de Erques: a) viaducto en construcción, b) viaducto finalizado.

migón en la fase de ejecución del tablero y cuelgue de las péndolas y, finalmente, los esfuerzos de servicio una vez superada la prueba de carga.

En este trabajo se describe el programa experimental realizado para determinar la dosificación del hormigón expansivo y el comportamiento frente al deslizamiento del sistema tubo de acero-hormigón, así como la instrumentación in situ de los arcos del puente para verificar dicho comportamiento frente a la expansión del hormigón. La finalidad de esta instrumentación ha sido determinar las deformaciones que se producen en los tubos de los arcos metálicos durante el proceso de llenado con un hormigón autocompactante de carácter expansivo y posterior endurecimiento, así como comprobar si los resultados son acordes a los determinados en la investigación experimental previa realizada con tubos de la misma relación diámetro/espesor utilizada en el viaducto.

\section{2.}

\section{HORMIGÓN EXPANSIVO}

Una importante innovación aplicada en el diseño de la estructura ha sido el empleo de hormigón expansivo como relleno de los tubos de los arcos. El objetivo de la expansividad es el de generar una presión de confinamiento permanente, que asegu-

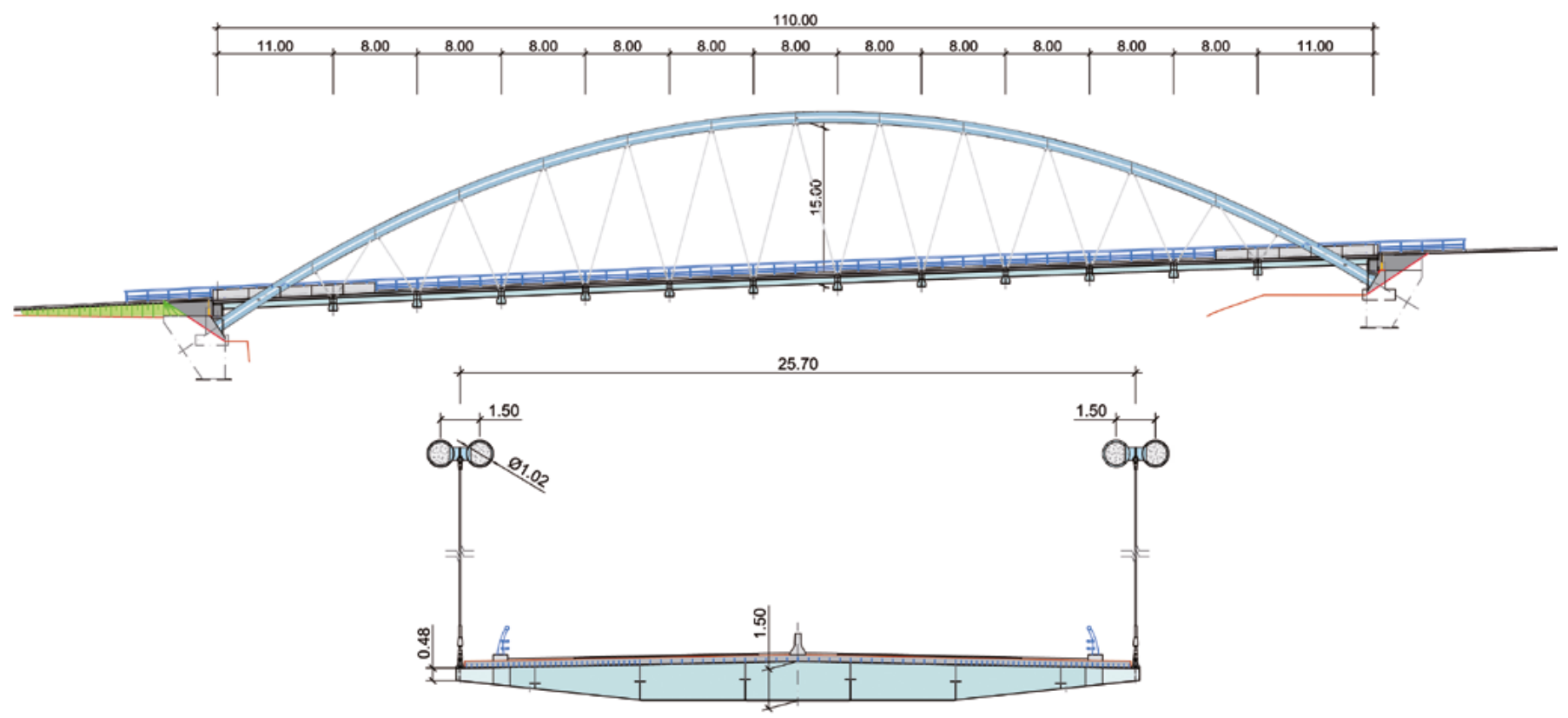

Figura 2. Esquema estructural en alzado y sección del viaducto. 

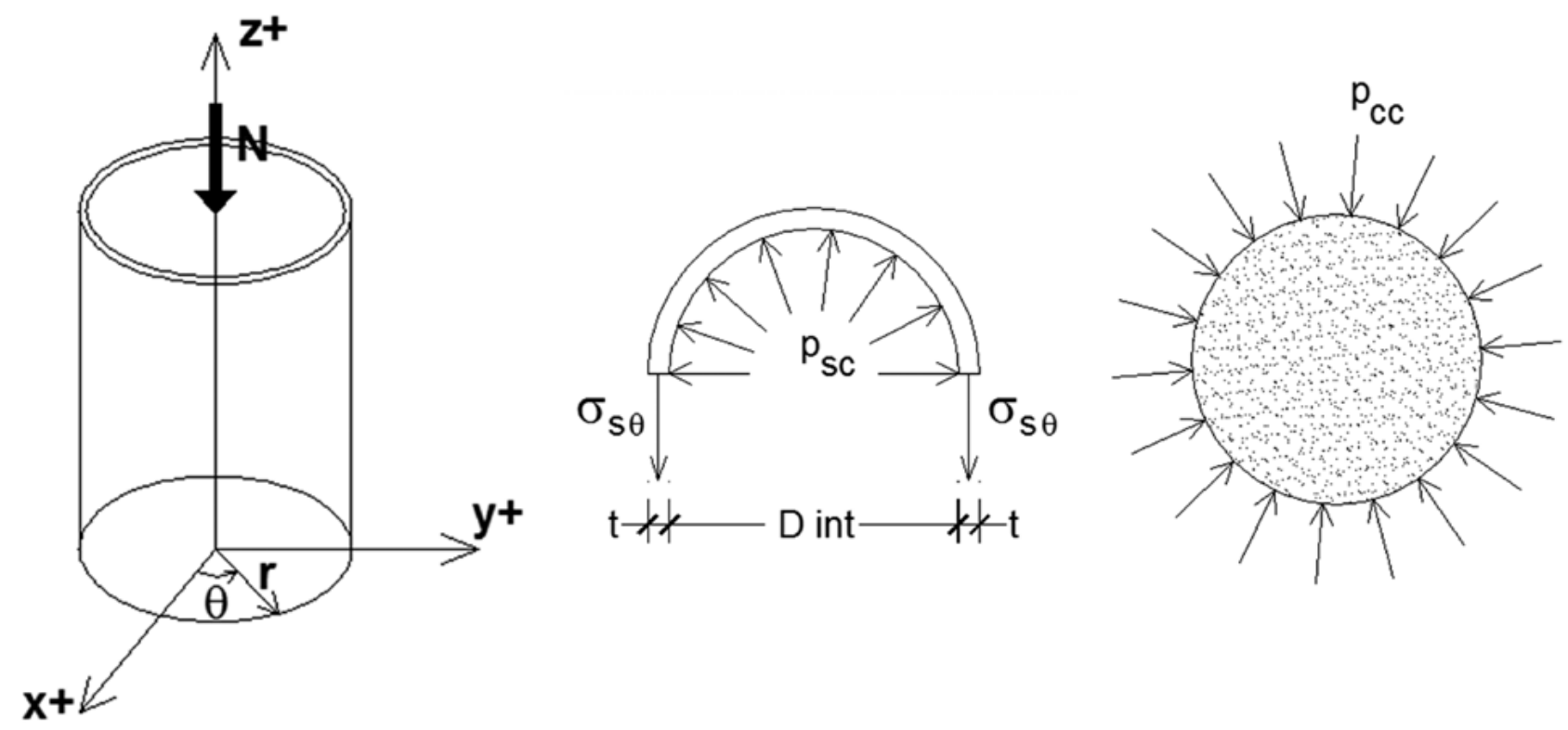

Figura 3. Tensiones en la sección transversal del tubo de acero y del núcleo de hormigón.

re el contacto entre hormigón y acero, y que, por tanto, haga factible la transmisión de esfuerzo rasante entre ambos materiales sin la utilización de conectores. Para mantener dicha presión de confinamiento, la expansividad deberá contrarrestar los efectos de la retracción del propio relleno y del calentamiento exterior del tubo, efectos que pueden provocar la separación de la interfase entre ambos.

A continuación se recogen las ecuaciones fundamentales que rigen los sistemas mixtos tubo-hormigón, indicando, aunque sea someramente, las bases de su dimensionamiento y comprobación. Estas ecuaciones permiten estimar la presión de confinamiento generada, así como las tensiones y deformaciones que dicha presión induce en el tubo y el hormigón, ecuaciones de la (1) a (6). La distribución de tensiones de la sección compuesta frente a flexión y axil se puede estimar con las ecuaciones (7) y (8).

A nivel de predimensionamiento sirven, por una parte, para determinar la presión de confinamiento y las deformaciones asociadas confinadas a partir de tanteos con una deformación libre asumida para el hormigón, de forma que se garantice el valor de la tensión de deslizamiento necesaria y, por otra, para estimar las tensiones normales de la sección compuesta frente a las solicitaciones de flexocompresión. El conocimiento de las deformaciones teóricas de expansión es también un punto de partida $\mathrm{u}$ orden de magnitud para controlar las deformaciones que deben medirse en los ensayos y, en consecuencia, estimar el porcentaje de aditivo expansor necesario.

Desde el punto de vista teórico, una forma de abordar el cálculo de las deformaciones y tensiones generadas en ambos materiales consiste en plantear las ecuaciones de equilibrio de fuerzas y la compatibilidad de deformaciones en la superficie de contacto, figura 3.

En general, para un axil exterior $\mathrm{N}$ actuando sobre un tubo mixto de acero - hormigón junto a una expansión del hormi- gón y una dilatación térmica de los materiales, la ecuación de equilibrio según el eje vertical z es la ecuación (1).

$\sigma_{s z} A_{s}+\sigma_{c z} A_{c}=N$

Donde $\sigma_{s z}$ y $\sigma_{c z}$ son la tensión vertical en el acero y en el hormigón, respectivamente, que actúan sobre un área de acero $A_{s}$ y un área de hormigón $A_{c}$.

En la sección transversal, la ecuación de equilibrio del tubo es la ecuación (2).

$p_{s c}=2 \sigma_{\mathrm{s \theta}} t / D_{i n t}$

Siendo $\sigma_{\mathrm{s} \theta}$ la tensión en la dirección $\theta$ en el acero, $D_{\text {int }}$ el diámetro interior del tubo de acero y $t$ su espesor. La presión de confinamiento actuante sobre el núcleo de hormigón $\left(p_{c c}\right)$ es igual a la actuante sobre el tubo acero $\left(p_{s c}\right)$ pero de signo contrario.

Además, en cualquier punto de contacto tubo-hormigón deben de cumplirse las ecuaciones de compatibilidad de deformaciones:

$\sum \varepsilon_{s z}=\sum \varepsilon_{c z}$

$\sum \varepsilon_{s \theta}=\sum \varepsilon_{c \theta}$

Cuando solo se considera el efecto de la deformación libre del hormigón, la ecuación queda reducida a las expresiones (5) y (6).

$\varepsilon_{s z}^{0}=\varepsilon_{c z}^{0}+\varepsilon_{c l}$

$\varepsilon_{s \theta}^{0}=\varepsilon_{c \theta}^{0}+\varepsilon_{c l}$

Donde $\varepsilon_{s z}^{0}$ y $\varepsilon_{c z}^{0}$ son las deformaciones según el eje $z$ del acero y del hormigón, respectivamente, debido a las tensiones longitudinales y transversales que se generan, $\varepsilon_{s \theta}^{0}$ y $\varepsilon_{c \theta}^{0}$ las deformacio- 


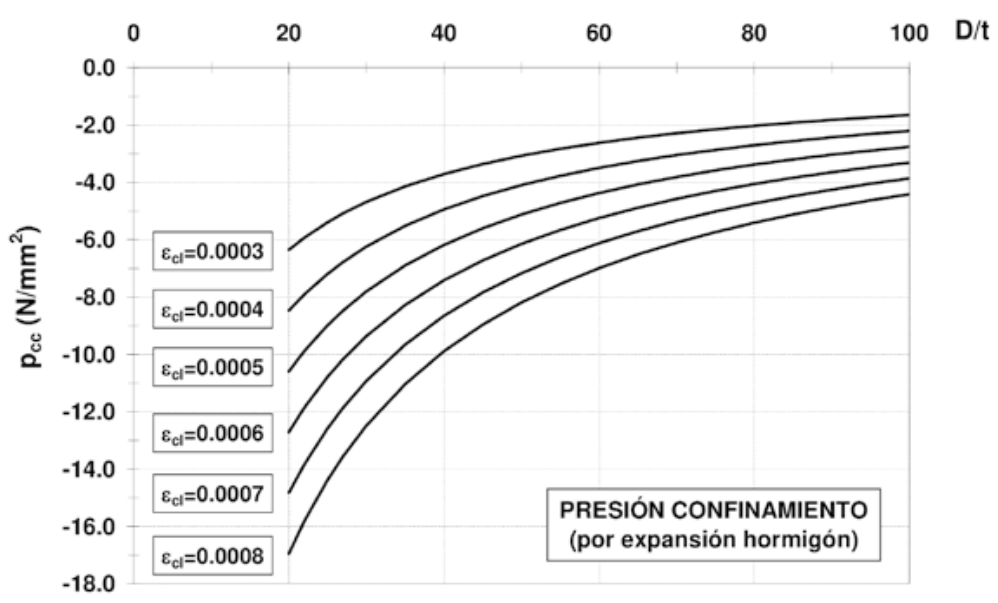

Figura 4. Presión de confinamiento para distinto $\varepsilon_{c l}$ y $\mathrm{D} / \mathrm{t}\left(f_{c}=50 \mathrm{~N} / \mathrm{mm}^{2}\right.$ y $\left.v_{c}=0,20\right)$.

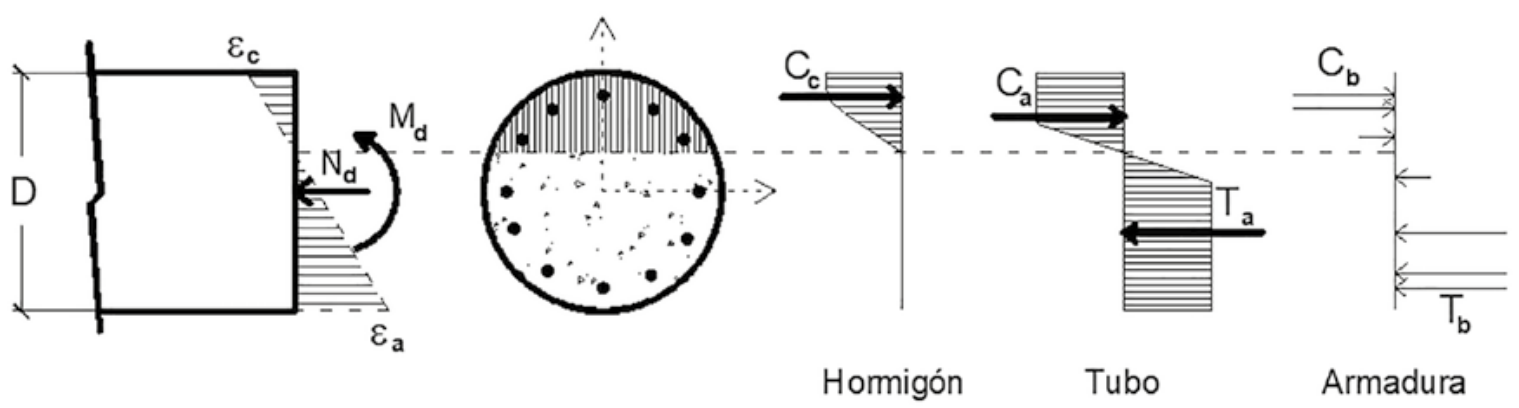

Figura 5. Distribución de tensiones y deformaciones en la sección transversal.

nes según el eje $\theta$ y $\varepsilon_{c l}$ la propia deformación libre considerada para el hormigón.

Estas ecuaciones son válidas para estudiar el efecto de una deformación de expansión libre del núcleo de hormigón $\left(\varepsilon_{c l}\right)$. A partir de estas ecuaciones, expresando las deformaciones en función de las tensiones, se pueden determinar la tensión en el hormigón, en el acero y la presión de confinamiento.

Cuando existen deformaciones de origen térmico, estas deben incluirse en las ecuaciones de compatibilidad, tanto para el acero como para el hormigón. Las deformaciones libres debidas a la temperatura se pueden estimar con las expresiones: $\varepsilon_{s t}=\alpha_{s} \Delta_{t s} \mathrm{y}$ $\varepsilon_{c t l}=\alpha_{c} \Delta_{t c}$, siendo $\alpha_{s}$ y $\alpha_{c}$ los coeficientes de dilatación térmica del acero y del hormigón, respectivamente y $\Delta_{t s}, \Delta_{t c}$ los incrementos de temperatura de cada material. Igual que se procede con las deformaciones anteriores puede hacerse con otras, como las deformaciones de retracción del núcleo de hormigón.

Un parámetro fundamental en el comportamiento resistente del sistema CFT es la relación entre el diámetro del tubo y su espesor (D/t $)$. Como se ha indicado, para unas mismas características del acero y del hormigón, las tensiones en ambos materiales debidas a la deformación de expansión del hormigón tienen el mismo valor si la relación $\mathrm{D} / \mathrm{t}$ permanece constante.

En la figura 4 se muestra como varía la presión de confinamiento del sistema mixto tubo-hormigón en función de la relación $\mathrm{D} / \mathrm{t}$ para distintos valores de deformación libre del hormigón. Como puede observarse, al aumentar la deformación de expansión del hormigón $\left(\varepsilon_{c l}\right)$, aumenta la presión de confinamiento, siendo esta mayor cuanto menor es la relación $\mathrm{D} / \mathrm{t}$.

Además de la incidencia de la relación $\mathrm{D} / \mathrm{t}$, las ecuaciones (1) a (6) permiten estudiar la influencia de diversas variables en la presión de confinamiento, entre otras, el axil soportado, que para una determinada deformación de expansión del hormigón, la presión de confinamiento disminuye al aumentar el nivel de solicitación axial de la sección; el valor de coeficiente de Poisson del hormigón, donde a mayor coeficiente de Poisson, mayor presión de confinamiento, si bien en los cálculos se adopta generalmente un valor igual a 0.2; o el módulo de elasticidad del hormigón, que a menor módulo de elasticidad, menor es la presión de confinamiento.

Respecto de la capacidad resistente de secciones CFT a flexocompresión, el análisis de la sección mixta frente a estas solicitaciones puede hacerse siguiendo un procedimiento similar al utilizado en el cálculo de secciones de hormigón armado sometidas a tensiones normales producidas por la solicitación exterior de un axil y un momento flector. La figura 5 muestra la geometría de la sección transversal, así como la distribución de tensiones y deformaciones en los distintos materiales que pueden conformar el sistema para esfuerzos de flexocompresión (se ha representado un caso genérico en el que puede haber armadura longitudinal en el hormigón). 
Por equilibrio de fuerzas y momentos en la sección, es posible estimar el valor de axil y momento último resistido. Las ecuaciones de equilibrio de fuerzas y momentos se obtienen igualando las resultantes de tensiones del hormigón y del acero, función de las deformaciones, con los esfuerzos exteriores aplicados (axil $N$ y momento $M$ ). Para una sección de canto $h$ igual al diámetro exterior del tubo, figura 6, las ecuaciones de equilibrio son las siguientes:

$$
\begin{aligned}
& N=\Sigma \mathrm{A}_{s i} \sigma_{s i}+\int_{h} \sigma_{c}(y) b(y) d y+\int_{h} \sigma_{a}(y) e(y) d y \\
& M=\Sigma \mathrm{A}_{s i} \sigma_{s i} y_{s i}+\int_{h} \sigma_{c}(y) b(y) y d y+\int_{h} \sigma_{a}(y) e(y) y d y
\end{aligned}
$$

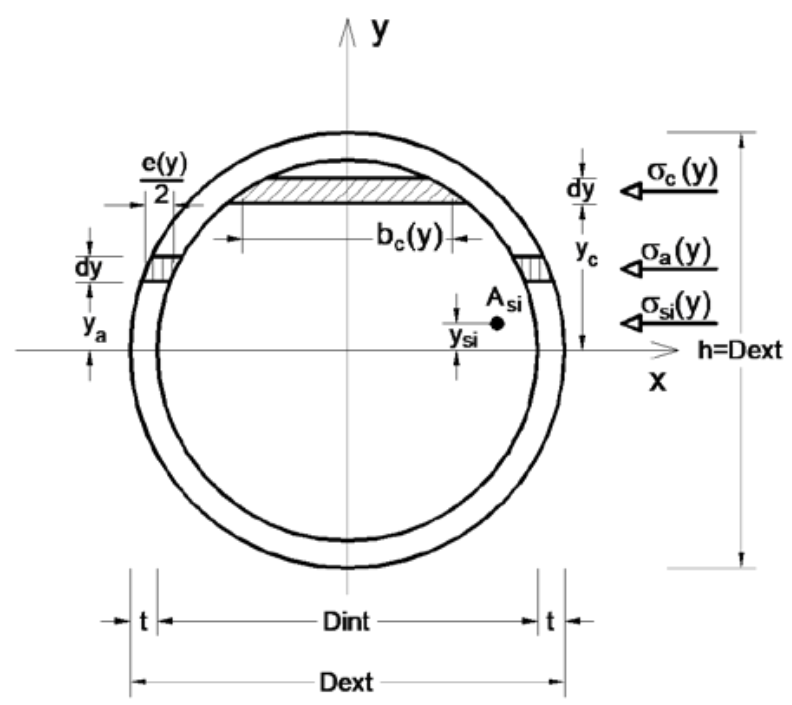

Figura 6. Sección transversal del tubo mixto acero-hormigón.

El hormigón del núcleo está confinado por lo que se puede considerar en el análisis un incremento de la resistencia del hormigón y de su deformación última. En el Código Modelo 2010 [16] se recoge una formulación explícita para estimar la resistencia a compresión del hormigón confinado en función de la tensión de confinamiento y de la resistencia del hormigón sin confinar. El efecto del confinamiento incrementa la resistencia del hormigón, que a su vez se traduce en una mayor presión de confinamiento. Este incremento puede evaluarse entre un $5 \%$ para relaciones altas de $\mathrm{D} / \mathrm{t}$ y un $14 \%$ para relaciones bajas de $\mathrm{D} / \mathrm{t}$.

Entre las variables que deben estudiarse está, entre otras, la relación entre el diámetro exterior del tubo y su espesor $(\mathrm{D} / \mathrm{t})$. Esta relación es un factor determinante en la capacidad resistente de la sección. Para un mismo tipo de hormigón y tipo de acero, secciones con la misma relación $\mathrm{D} / \mathrm{t}$ tienen una misma capacidad resistente en términos de axil y momento normalizado $\left(\mathrm{N} / \mathrm{N}_{0}\right.$ y $\left.\mathrm{M} / \mathrm{M}_{0}\right)$, siendo $\mathrm{M}_{0}$ la capacidad resistente a flexión con axil nulo y $\mathrm{N}_{0}$ la capacidad resistente a compresión con momento nulo.

De forma análoga tubos con el mismo tipo de acero estructural y hormigón y la misma relación $\mathrm{D} / \mathrm{t}$ tienen el mismo diagrama axil momento normalizado $\left(\mathrm{N} / \mathrm{N}_{0}\right.$ y $\left.\mathrm{M} / \mathrm{M}_{0}\right)$ si la cuantía geometría de armado interior es la misma.
3.

\section{PROGRAMA EXPERIMENTAL}

Se ha desarrollado un programa experimental que ha permitido evaluar los parámetros fundamentales que rigen el comportamiento de este tipo de sistema mixto.

Este programa ha consistido: en obtener unas dosificaciones de hormigón autocompactante que generasen una expansión cercana a la deseada; realizar ensayos a deslizamiento con tubos de relación diámetro/espesor similar a los proyectados para los arcos del viaducto; así como llevar a cabo una instrumentación in situ de los arcos para evaluar las deformaciones y temperaturas que se producen en los tubos durante el hormigonado, fraguado y endurecimiento del hormigón.

El análisis estructural ha evaluado el rasante en los arcos del viaducto. Este rasante es variable a lo largo de la luz, estimándose la tensión rasante máxima de cálculo en $0.29 \mathrm{~N} / \mathrm{mm}^{2}$. Este valor es uno de los requisitos fundamentales para la investigación experimental, traducido en unos valores de la presión de confinamiento y de las deformaciones asociadas del hormigón, libre y restringida.

\subsection{Ensayos de caracterización}

Se ha realizado un amplio plan de ensayos, desarrollado en varias fases, para determinar una dosificación óptima del hormigón que cumpliera con los requisitos de expansión necesaria, de resistencia y que fuera autocompactable.

En una primera fase se han estudiado como variables diversos tipos de cementos (42.5 y 52.5) y de superplastificantes, así como dos tipos de productos de expansión, que se probaron con distintos porcentajes dentro de los límites recomendados por el fabricante. Cada dosificación se ha caracterizado en estado freso, determinando la fluidez, densidad y contenido de aire, y en estado endurecido, evaluando su resistencia y expansión.

Además, para cada dosificación se realizaron dos ensayos con probetas para evaluar la deformación libre y otras dos probetas para determinar la deformación restringida. Los ensayos de expansión libre se han realizado según el método de ensayo recogido en la norma ASTM C157 [17]. Los ensayos para estimar la expansión restringida se han hecho según el método de ensayo recogido en la norma ASTM C878 [18].

Para asegurar la autocompactabilidad del hormigón de relleno de los tubos, se han realizado ensayos de escurrimiento, realizados según UNE-EN 12350-8 [19], comprobando que se obtenían resultados homogéneos y acordes con los requisitos que se recogen en el Anejo 17 de la EHE-08 [13].

En una segunda fase se procedió al ajuste y optimización de los tipos de cementos y porcentajes de los aditivos expansivos. Se empezaron a realizar, además, varios ensayos con tubos pequeños y relación $\mathrm{D} / \mathrm{t}$ similar al empleado en obra, cuyo objeto era comprobar el orden de magnitud de las deformaciones circunferenciales que se generaban. Con varios de estos tubos, de diferentes alturas, se hicieron unos ensayos preliminares de deslizamiento.

Después de varias dosificaciones y pruebas, se determinó la dosificación de estudio que mejor se adaptaba a las exigencias requeridas. Se eligió un cemento CEM I- 52.5 $\mathrm{R}$ y un aditivo de expansión tipo DRY, con un porcentaje de dosificación comprendido entre el $8 \%$ y el $11 \%$ del contenido de cemento. Con 


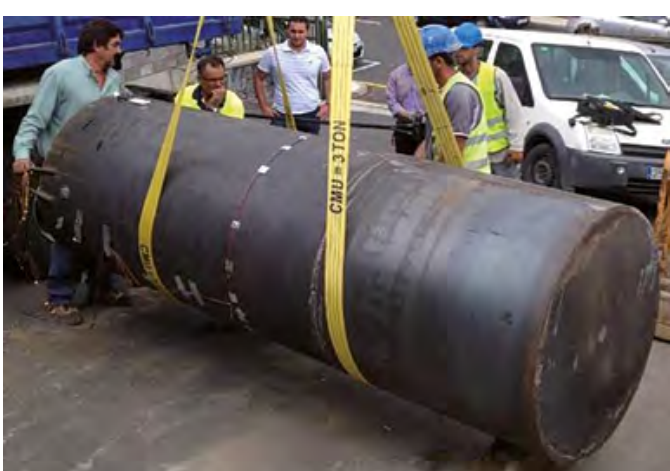

a)

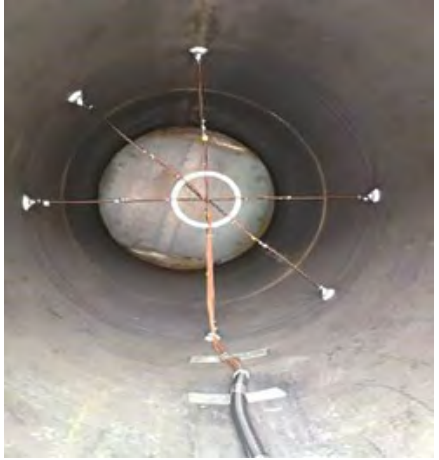

b)

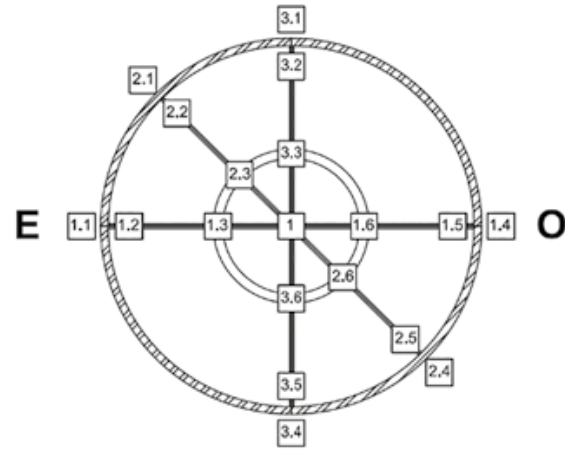

c)

Figura 7. Tubo in situ e instrumentación interna: a) tubo, b) instrumentación interna, c) disposición de termopares.

estos porcentajes se obtenían deformaciones lineales libres comprendidas, mayoritariamente, entre el $0.55 \%$ y el $0.75 \%$, que se consideraron adecuadas para cumplir, en principio, las exigencias de proyecto. Las deformaciones circunferenciales medidas en los tubos estaban comprendidas entre $0.45 \%$ y el $0.65 \%$, y las tensiones medias de deslizamiento entre $0.55 \mathrm{~N} / \mathrm{mm}^{2}$ y $0.95 \mathrm{~N} / \mathrm{mm}^{2}$. En el apartado 3.4 se recoge una estimación de estas tensiones obtenidas de ensayos de deslizamiento para tubos con distintas condiciones de temperatura.

Teniendo en cuenta las variables analizadas en las dos fases preliminares, se han preparado más de 30 dosificaciones tipo. En la tercera fase se concretó el porcentaje final de aditivo expansivo a utilizar y se realizaron los ensayos de deslizamiento que se recogen en el apartado 3.2.

En esta tercera fase se han realizado una serie de ensayos para obtener unas dosificaciones de hormigón autocompactante que generen una expansión cercana a la deseada, estimada entre $0.4 \%$ y $0.5 \%$. Como dosificaciones de estudio de los hormigones se han considerado aquellas con un contenido de cemento del orden de $480 \mathrm{~kg} / \mathrm{m}^{3}$, una relación agua cemento de 0.4 , una dosis de aditivo expansivo tipo DRY en torno al $10 \%$ del contenido de cemento y aditivos superplastificantes con una dosificación comprendida entre el $1.6 \%$ y el 1.8\%, obteniendo resistencias del orden de $50 \mathrm{~N} / \mathrm{mm}^{2}$.

La dosificación final empleada por $\mathrm{m}^{3}$ fue la siguiente: 485 $\mathrm{kg}$ de cemento CEM I - $52.5 \mathrm{R} ; 230 \mathrm{~kg}$ de agua; $489 \mathrm{~kg}$ de grava 6/12; 692 de arena 0/6; $337 \mathrm{~kg}$ de arena 0/3; $8.2 \mathrm{~kg}$ de superplastificante y $48.5 \mathrm{~kg}$ de DRY Dl C.

El superplastificante añadido fue GLENIUM SKY 549. El porcentaje de aditivo expansivo empleado está dentro de las especificaciones del fabricante. Dicho porcentaje podría considerarse elevado para hormigones sin confinar, pero resulta apto para la finalidad propuesta (confinamiento del hormigón en el interior del tubo) con objeto de alcanzar los requerimientos de diseño.

\subsection{Ensayos de deslizamiento}

Para estudiar los tubos rellenos de hormigón se han realizado ensayos de deslizamiento a temperatura ambiente y con calentamiento exterior uniforme y diferencial, para evaluar la influencia de la temperatura en la resistencia a deslizamiento del núcleo de hormigón.
Previo a estos ensayos, y con el objeto de ajustar los valores de la acción térmica, se instaló un tubo de acero relleno de hormigón en Tejina de Guía (Tenerife). El tubo se colocó con la misma orientación que llevaría el futuro puente. Para el registro de temperaturas se colocaron 19 termopares en distintos puntos del núcleo de hormigón y en el tubo de acero. En una zona exterior al tubo se colocó otro termopar para registrar la temperatura ambiente. Todos los termopares instalados son tipo $\mathrm{T}$ y van protegidos contra la humedad y esfuerzos mecánicos. En la figura 7 se puede ver la disposición de los termopares en una sección del tubo.

De las medidas que se registraron se puede destacar que las mayores temperaturas se obtuvieron en los termopares 3.1 y 3.2 , con valores en torno a $\operatorname{los} 65^{\circ} \mathrm{C}$. La diferencia de temperatura media entre las medidas exteriores del tubo de acero y el interior del hormigón (termopar 1) fue de unos $30^{\circ} \mathrm{C}$ para el periodo de mayor soleamiento. La diferencia de temperaturas entre caras opuestas del eje vertical fue del orden de $28^{\circ} \mathrm{C}$.

Conocidos los valores de acción térmica, el programa experimental consistió en el ensayo de 9 tubos de acero de 1264 $\mathrm{mm}$ de altura, $316 \mathrm{~mm}$ de diámetro interior y $4 \mathrm{~mm}$ de espesor, figura 8. El diámetro del tubo de ensayo es aproximadamente $1 / 3$ del diámetro del tubo de la obra real y la longitud de $1264 \mathrm{~mm}$ es suficiente para poder movilizar todo el cilindro de hormigón frente al deslizamiento con los medios disponibles en el laboratorio. La relación D/t de los tubos ensayados, parámetro fundamental en el comportamiento resistente del sistema CFT, es similar a la de los arcos del viaducto, por lo que el factor de escala de los ensayos se amortigua, permitiendo que los resultados puedan ser comparados.

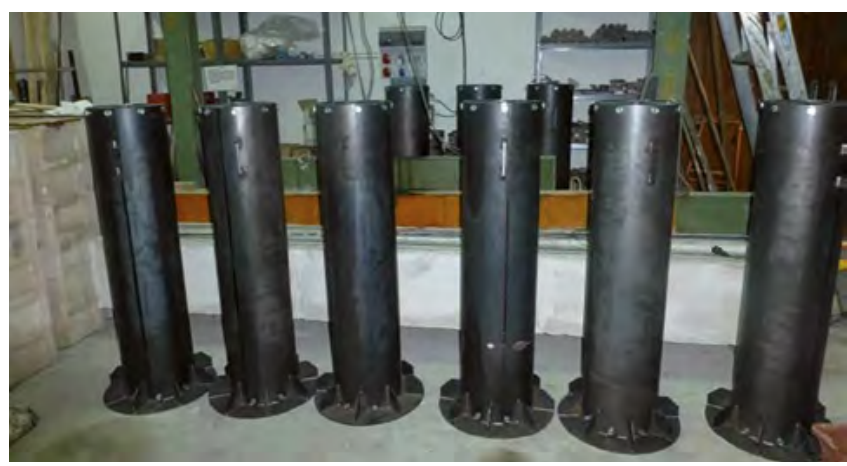

Figura 8. Vista general de varios de los tubos metálicos. 


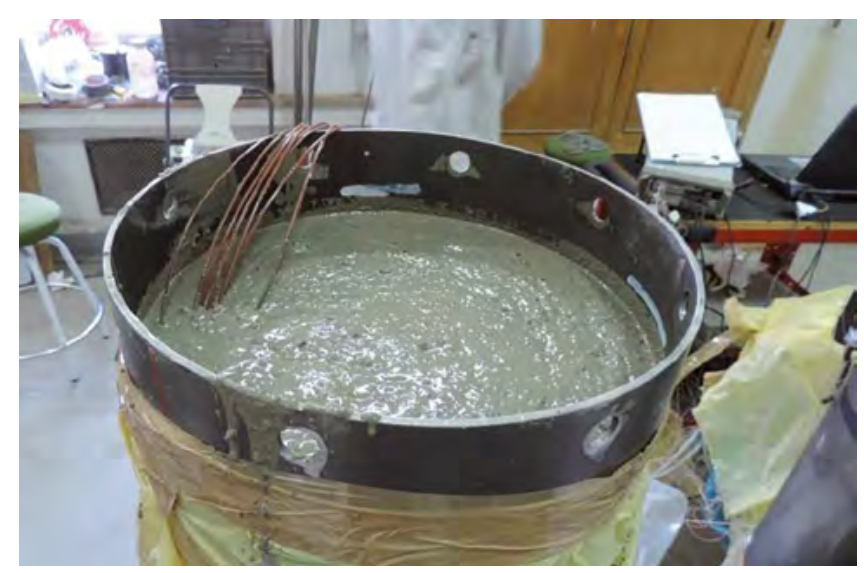

Figura 9. Tubo hormigonado.

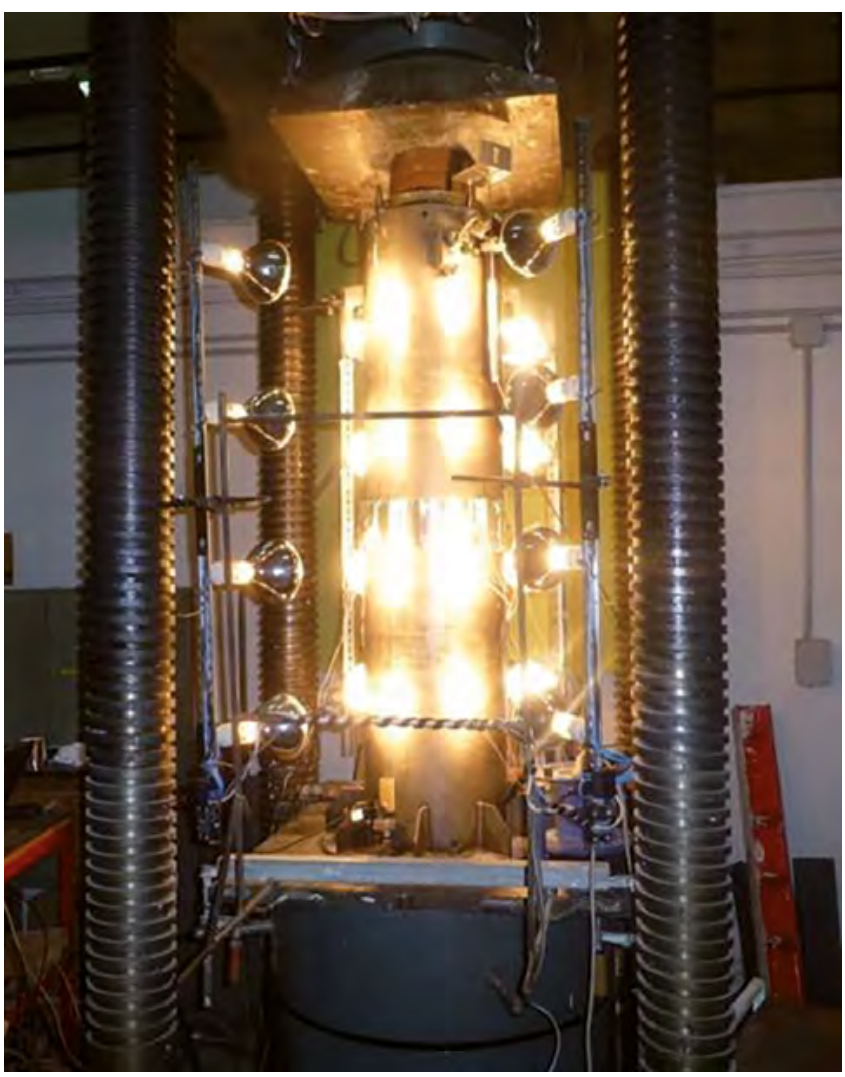

Figura 11. Ensayo de un tubo con calentamiento uniforme.

Los tubos se llenaron de hormigón con un 10\% de aditivo expansivo, figuras 9 y 10.

Los ensayos realizados fueron los siguientes:

- Tres tubos a temperatura ambiente.

- Tres tubos sometidos a un calentamiento exterior uniforme, entre $63^{\circ} \mathrm{C}$ y $65^{\circ} \mathrm{C}$ en la superficie, manteniéndose el núcleo de hormigón a $32^{\circ} \mathrm{C}$.

- Tres tubos sometidos a un calentamiento diferencial, con una diferencia de temperatura entre un lado y el otro del tubo de unos $32^{\circ} \mathrm{C}$, algo mayor que la obtenida en el ensayo in situ. La temperatura del núcleo de hormigón se mantuvo entre $32^{\circ} \mathrm{C}$ y $34^{\circ} \mathrm{C}$ en los distintos ensayos.

Los tubos se ensayaron a deslizamiento pasados más de 28 días de su hormigonado. Los ensayos se realizaron con una prensa

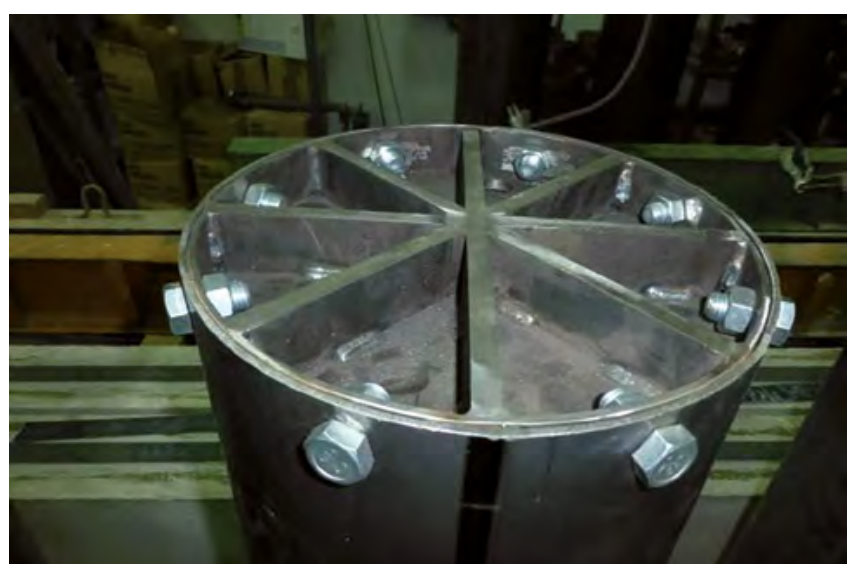

Figura 10. Tapa superior atornillada.

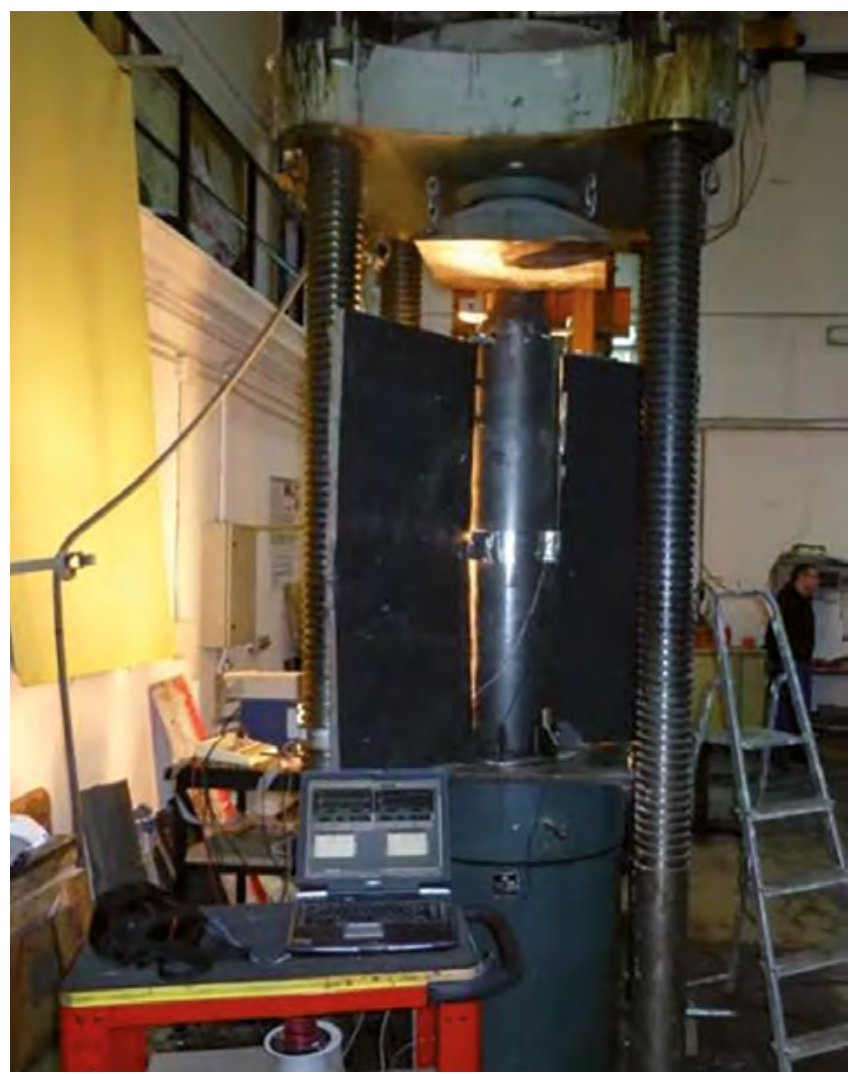

Figura 12. Ensayo de un tubo con calentamiento diferencial.

ICON de 1000 toneladas de capacidad (10 $000 \mathrm{kN})$, figuras 11 y 12. Los ensayos consintieron en aplicar con la prensa una carga axial con objeto de hacer deslizar el núcleo de hormigón respecto del tubo de acero.

\subsection{Instrumentación de los tubos de ensayo}

Para el registro de temperatura, se han colocado termopares dentro del hormigón para medir la temperatura que se desarrollaba tanto en el proceso de fraguado como durante la fase de calentamiento.

Los termopares se han colocado en la sección situada a la mitad de la altura del tubo. De estos termopares, uno se colocó en el centro de la sección de hormigón, disponiendo los otros circunferencialmente, en diámetros perpendiculares, a distan- 
TUBOS 7, 8 y 9. Deformación circunferencial media de la sección central

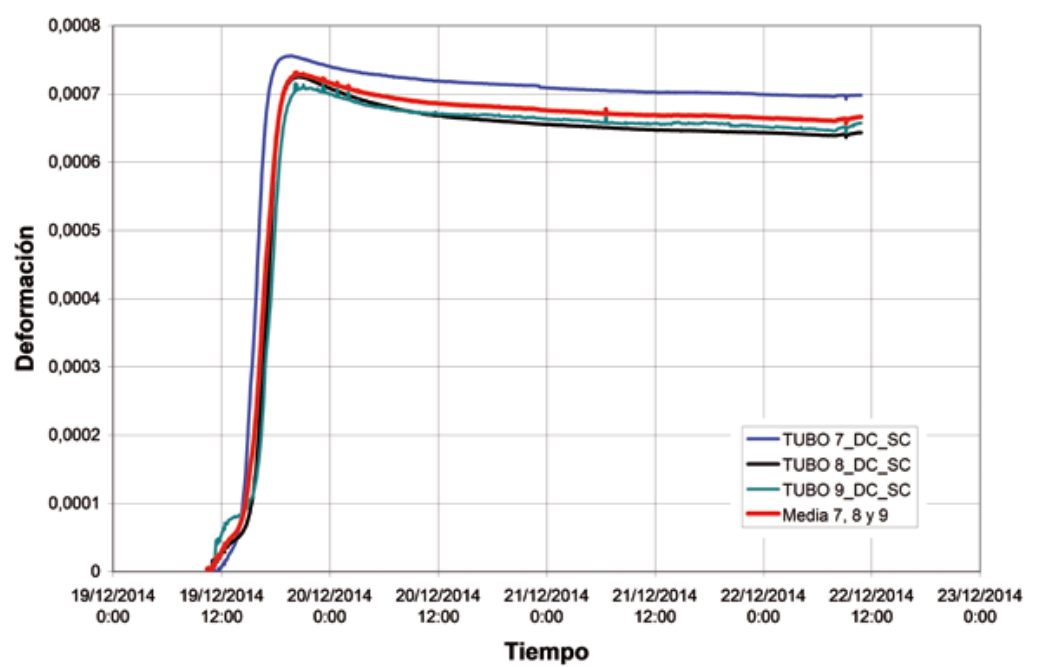

\begin{tabular}{|c|c|c|}
\hline Tubo & $\begin{array}{l}\text { Deformación } \\
\text { circunferencial en } \\
\text { sección central }\end{array}$ & $\begin{array}{l}\text { Deformación } \\
\text { media }\end{array}$ \\
\hline 1 & $6,11 \cdot 10^{-4}$ & \multirow{3}{*}{$6,36 \cdot 10^{-4}$} \\
\hline 2 & $6,76 \cdot 10^{-4}$ & \\
\hline 3 & $6,21 \cdot 10^{-4}$ & \\
\hline 4 & $6,60 \cdot 10^{-4}$ & \multirow[t]{3}{*}{$6,67 \cdot 10^{-4}$} \\
\hline 5 & $6,37 \cdot 10^{-4}$ & \\
\hline 6 & $7,03 \cdot 10^{-4}$ & \\
\hline 7 & $6,98 \cdot 10^{-4}$ & \multirow[t]{3}{*}{$6,63 \cdot 10^{-4}$} \\
\hline 8 & $6,41 \cdot 10^{-4}$ & \\
\hline 9 & $6,52 \cdot 10^{-4}$ & \\
\hline
\end{tabular}

Figura 13. Deformaciones circunferenciales registradas en los tubos: a) Gráfica Deformaciones circunferenciales registradas en los tubos 7, 8 y 9, b) Tabla con las deformaciones de los tubos.

cias de un tercio y dos tercios del radio.

Para la medida de desplazamientos, se han colocado dos transductores en la parte superior tipo LVDT y otro en la parte inferior. Durante el ensayo se registraba la carga aplicada, así como los movimientos relativos entre hormigón y acero.

Para el calentamiento de los tubos se han empleado 16 lámparas OMRON de $250 \mathrm{~W}$ controladas con micro Arduino conectado a relés de estado sólido. En los ensayos con calentamiento diferencial se protegió la mitad del tubo con paneles aislantes.

\subsection{Resultados}

En cuanto al calor de hidratación, la temperatura media alcanzada en el centro del hormigón durante la hidratación fue del orden de $73^{\circ} \mathrm{C}$.

Respecto a las deformaciones circunferenciales de expansión, los resultados obtenidos fueron similares en todos los tubos, registrándose a las 72 horas una deformación media del orden de $6.55 \cdot 10^{-4}$. La figura 13 recoge las deformaciones registradas en los tubos 7, 8 y 9 .

Respecto a los ensayos de deslizamiento a temperatura ambiente, la tensión media de deslizamiento ha sido de 0.846 $\mathrm{N} / \mathrm{mm}^{2}$, obtenida dividiendo la fuerza aplicada por la superficie de contacto entre hormigón y tubo. En los ensayos de deslizamiento con calentamiento uniforme, la tensión media de deslizamiento ha sido de $0.716 \mathrm{~N} / \mathrm{mm}^{2}$ y en los ensayos con calentamiento diferencial, la tensión media de deslizamiento ha resultado de $0.663 \mathrm{~N} / \mathrm{mm}^{2}$.

La tensión media de deslizamiento del núcleo de hormigón disminuye un $15 \%$ para el caso de calentamiento uniforme y un $22 \%$ para el caso de calentamiento diferencial, respecto a la tensión media obtenida en los ensayos a temperatura ambiente de laboratorio. En el caso más desfavorable, la tensión rasante obtenida en el ensayo es mayor que dos veces la máxima tensión rasante en el estado límite último en los arcos del viaducto.
4.

\section{INSTRUMENTACIÓN DE LOS TUBOS DE LOS ARCOS DEL VIADUCTO SOBRE EL BARRANCO DE ERQUES}

Los arcos metálicos se fabricaron en tres trozos que se ensamblaron horizontalmente en la explanada próxima a uno de los estribos del puente. En esta situación se procedió a montar la instrumentación básica de las secciones de los arcos, que después se completó con los equipos de medida una vez dispuestos los arcos en su posición definitiva.

La figura 14 muestra un esquema de la sección del puente con los tubos instrumentados. En el arco 1 el tubo instrumentado fue el exterior y en el arco 2 fue el interior.

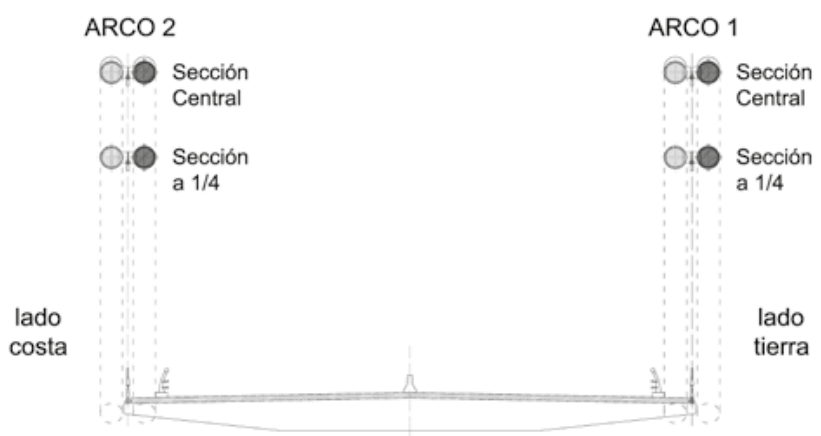

Figura 14. Sección del puente.

La instrumentación dispuesta para registrar las deformaciones longitudinales y circunferenciales y temperaturas en el exterior de los tubos, en 4 secciones, ha sido la siguiente:

\subsection{Medidas eléctricas}

Tubo del arco 1: Tubo exterior del arco.

En cada sección se ha utilizado un acondicionador QuantumX MX1615 de HBM para medir tanto la deformación como la 


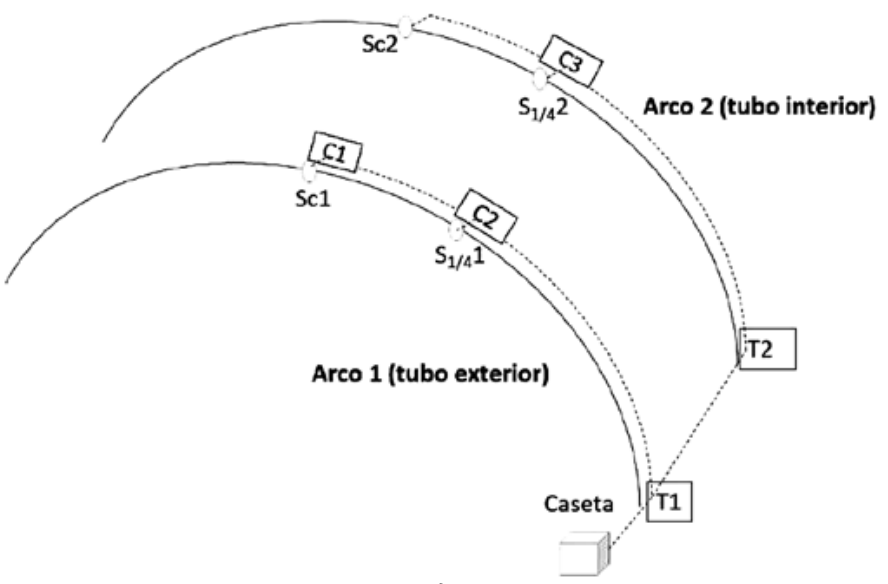

a)

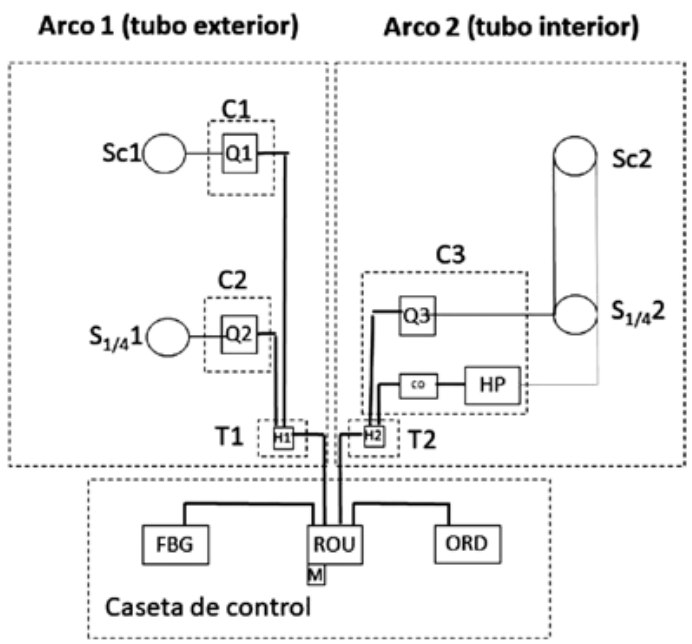

- Sc: sección clave

- $\mathrm{S}_{1 / 4}$ : sección a $1 / 4$

- C: caja

- T: terminador

- H: HUB

- Q: Equipo de medida Quantum

- HP: Eq. Hewlett Packard

- co: convertidor serie-tcpip

- FBG: interrogadorfibra óptica

- ROU: Router

- M: modem

- ORD: portátil

b)

Figura 15. Red local: a) Localización de la instrumentación en los arcos y b) Esquema general.

temperatura. La deformación se ha medido con sensores FCA3-11-1L de TML. Estos sensores son rosetas de dos galgas extensométricas dispuestas a $90^{\circ}$, de $3 \mathrm{~mm}$ de longitud y $120 \Omega$ de resistencia. La configuración eléctrica es en $1 / 4$ de puente a 4 hilos. La medida de la temperatura se llevaba a cabo con sensores PT100 de la casa TC (clase B, 4 hilos, encapsulado en silicona, flexibles, aislados con teflón y malla metálica).

\section{Tubo del arco 2: Tubo interior del arco.}

En la sección 1/4 se ha colocado un acondicionador QuantumX MX1615 que mide las deformaciones de las dos secciones del tubo del arco 2; utilizando para ello los mismos sensores y con la misma configuración que en el caso del tubo del arco 1. La temperatura se ha medido con termopares tipo $\mathrm{T}$ de la casa TC (modelo 3AY-T-10mm-3m-B50TX-3.0-dualwall hetshrink-insulated-cll, clase 1 , vaina de acero inoxidable de $3 \times 10 \mathrm{~mm}$, aislado con PFA) conectados a un equipo de adquisición de datos HP34970A de Hewlett Packard provisto de un módulo de 20 canales 34901A.

\subsection{Fibra óptica}

La sección a 1/4 del tubo 2 se ha instrumentado con sensores de fibra óptica tipo redes de Bragg para tener, en caso necesario, una alternativa a las medidas registradas. Se ha utilizado un array de $4 \mathrm{~m}$ de longitud con 4 sensores de $10 \mathrm{~mm}$ y en torno a $1550 \mathrm{~nm}$ de longitud de onda, montados en fibra monomodo 9/125 $\mu \mathrm{m}$. Para las conexiones se ha utilizado un equipo Fujikura FSM-18S y para la lectura un interrogador FBG Scan 800.

\subsection{Comunicaciones, software y tratamiento de datos}

Para el registro de medidas se ha montado una pequeña red local para conectar los equipos Quantum X y el interrogador fbg, con un ordenador portátil situado en el puesto de control. Para la conexión del equipo HP34970A se ha utilizado un convertidor RS232C-ethernet Expert EX9132. A los cuatro equipos encargados de las medidas eléctricas se les ha llevado un cable de acometida eléctrica para su alimentación. Todo el cableado, los equipos y los sensores se instalaron para trabajar a la intemperie.

La red local implementada es la que se indica en los esquemas de la figura 15. En el tubo del arco 1, tubo exterior, se colocó un equipo Quantum X en la sección central y otro en la sección a 1/4. En el tubo del arco 2, tubo interior, se dispuso otro equipo Quantum X en la sección a 1/4, para medir las deformaciones de las dos secciones instrumentadas y un equipo de adquisición de datos HP34970A con un convertidor RS232C para registrar las temperaturas. 
En la red local, los equipos de medida se conectaron mediante hubs a un router provisto de un modem 3G, que, a su vez, mediante sendos puertos libres se conecta al interrogador de fibra óptica y al ordenador.

El software utilizado para el control y la configuración de los equipos Quantum X ha sido el CatmanEasy de HBM. En cuanto al equipo de HP se ha utilizado el Agilent Bechlink Data Logger. Finalmente, para el manejo y control del interrogador se ha empleado el Illumisense de FBGS. El tratamiento de datos final para unificar y sincronizar todas las medidas se ha llevado a cabo con MatLab.

\subsection{Registros de deformaciones y temperaturas}

En cada tubo se han instrumentado dos secciones, una próxima a la clave del arco (sección clave o sección central) y otra situada a $30 \mathrm{~m}$ del extremo, que se denomina sección 1/4. En cada sección se han dispuesto cuatro puntos de medida y en cada punto se han instalado dos bandas extensométricas, una en dirección circunferencial y otra en dirección longitudinal, y un sensor de temperatura. La figura 16 recoge la situación del arco 1 en la explanada, mostrando lo que será su cara inferior en su posición definitiva en obra. En cada punto de medida se retira la pintura protectora del arco en una superficie pequeña para colocar las galgas y termopares, figura 17.

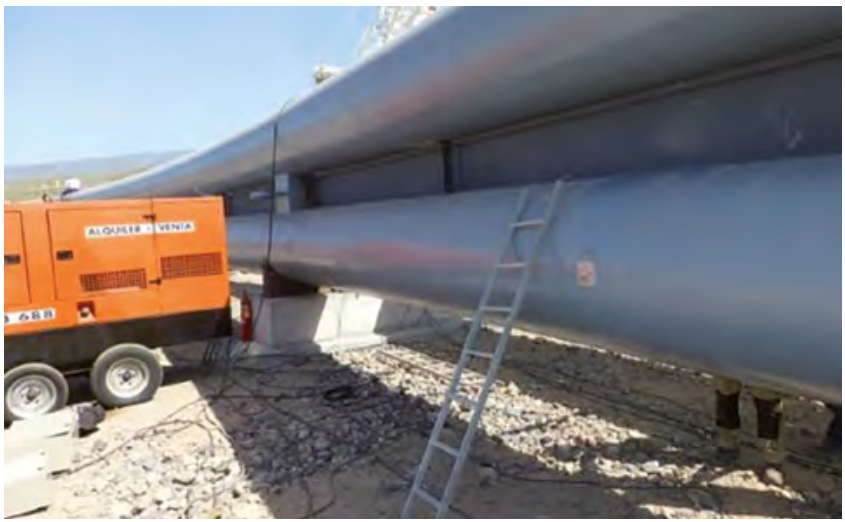

Figura 16. Arco 1 en explanada.

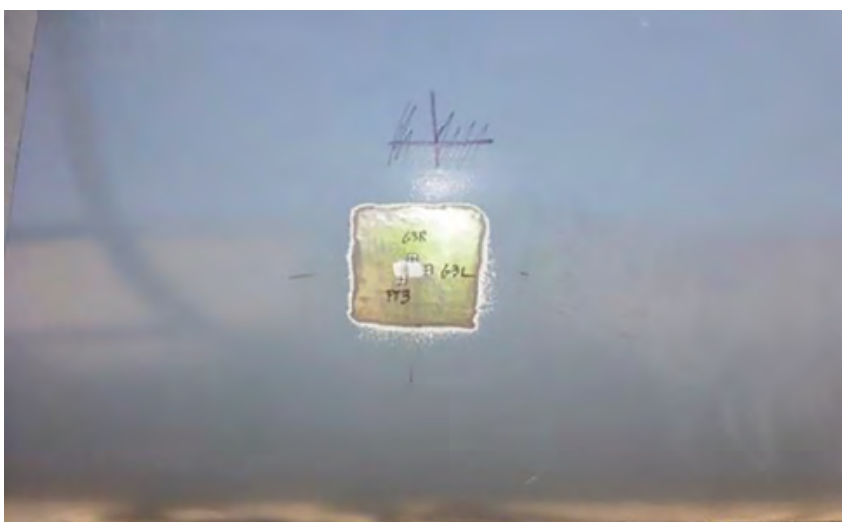

Figura 17. Detalle instrumentación.

Una vez colocadas las galgas en cada punto de medida, se procede al cableado de las mismas y a su protección con cajas registradoras y varias capas de aislante térmico, como se aprecia en la figura 18. Todo el cableado va protegido con tubos para intemperie. Una vez colocados los arcos en su posición definitiva mediante potentes grúas, figura 19, se montan los equipos de medida, figura 20, y se protegen dentro de las cajas dispuestas para este fin, figura 21 .

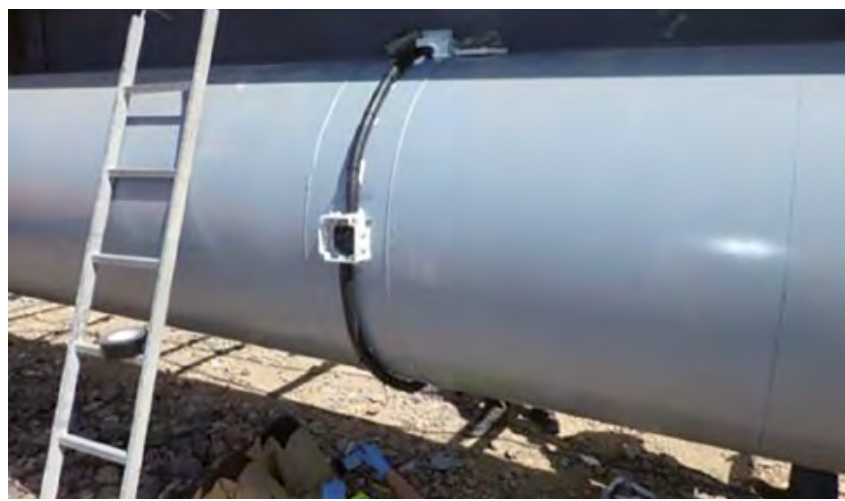

Figura 18. Colocación de la protección.

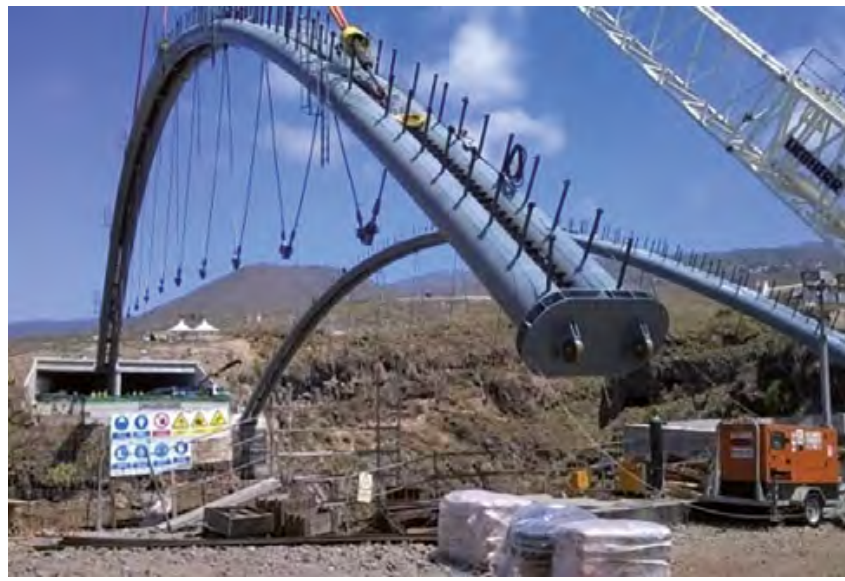

Figura 19. Colocación de Arcos en posición final

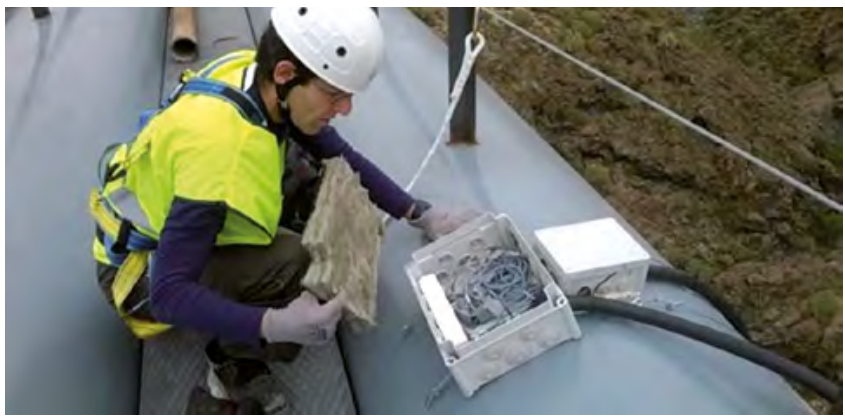

Figura 20. Colocación de equipos en arcos.

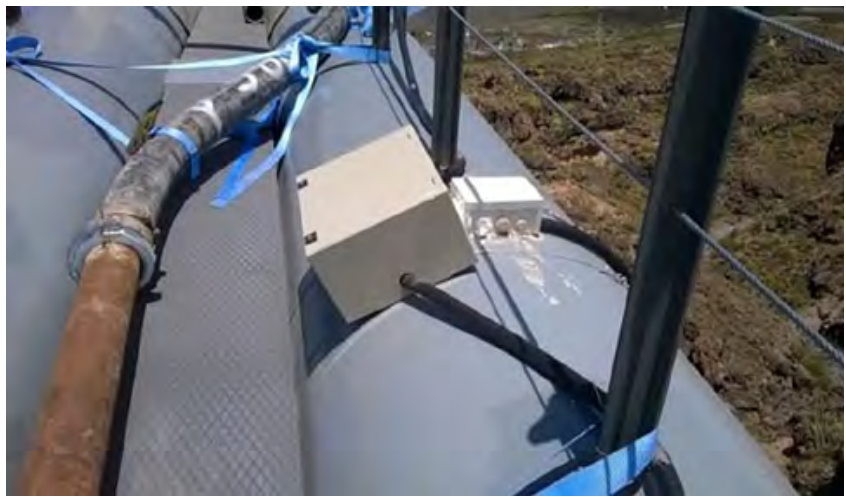

Figura 21. Cajas de protección. 

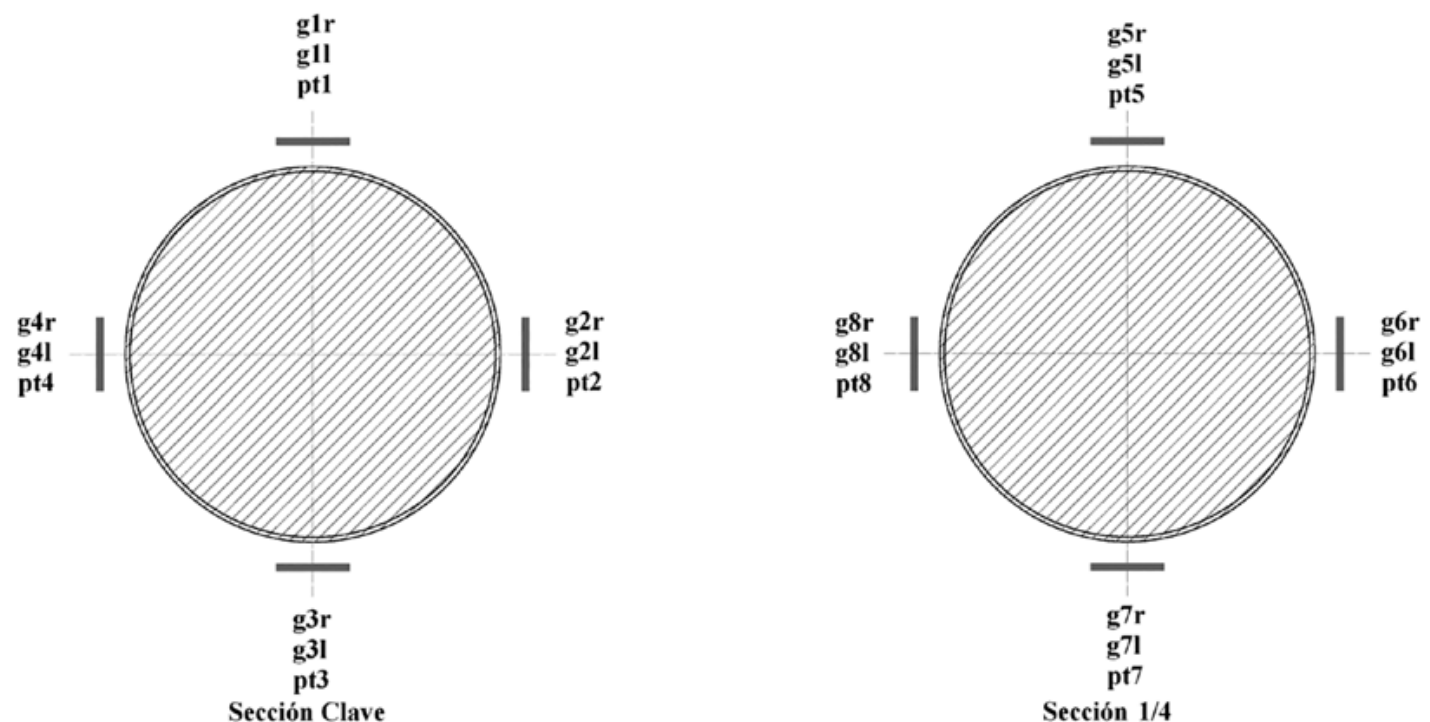

Figura 22. Denominación de galgas y termopares en secciones clave y 1/4 del tubo 1 .

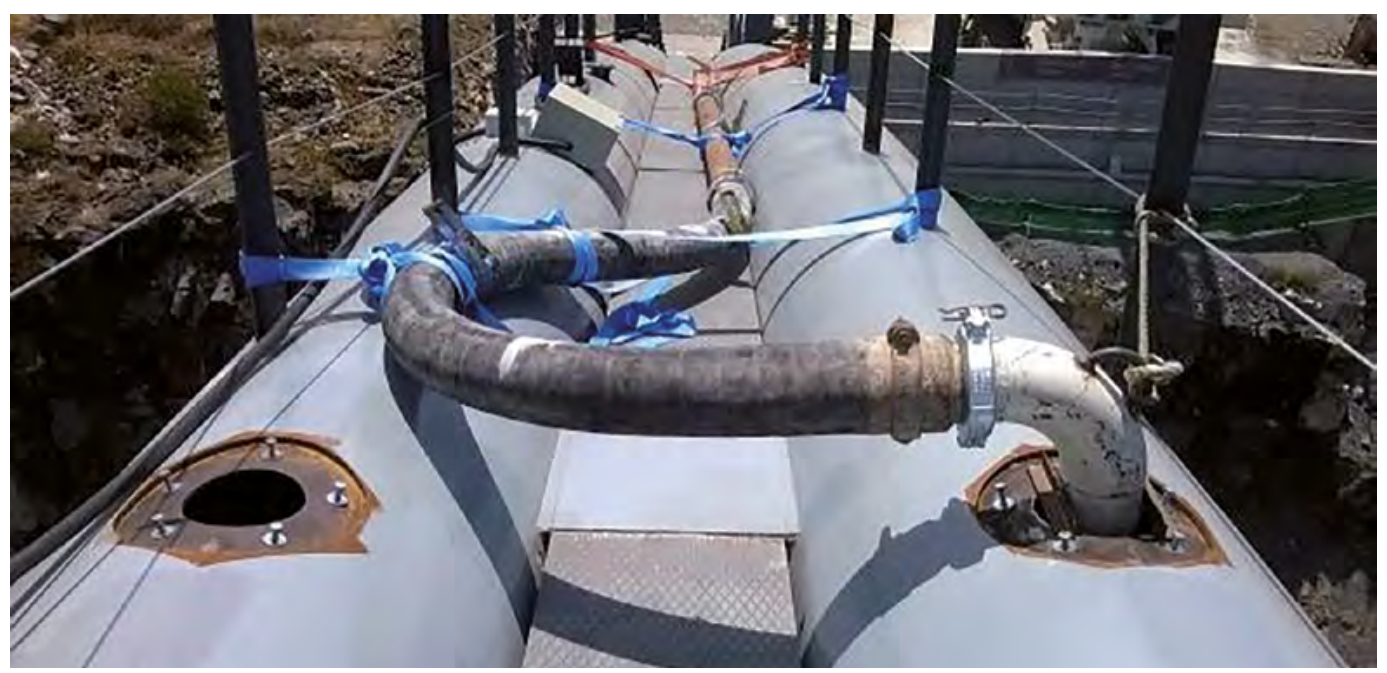

Figura 23. Hormigonado de arcos.

En la figura 22 se recoge el esquema de colocación de las galgas en las secciones clave y $1 / 4$ del tubo 1 . Para el tubo 2 se ha seguido el mismo esquema. Las señales de las galgas se corrigieron teniendo en cuenta su sensibilidad térmica (thermal output, hoja de datos del fabricante) si bien se ha constatado que esta corrección correspondiente a la temperatura máxima registrada fue un valor pequeño frente a los valores obtenidos. Durante un periodo de tiempo antes y después del hormigonado de los arcos se comprobó la estabilidad de las medidas.

Una vez instalados los equipos y probada la instrumentación los arcos quedaron preparados para su hormigonado.

Posteriormente, se procedió al relleno de los tubos con el hormigón autocompactante expansivo preparado en una planta próxima al viaducto. El hormigonado se efectuó mediante las ventanas y respiraderos dispuestos a diferentes alturas en una secuencia simétrica y ascendente, figura 23. El relleno de cada arco, en días distintos, se completó en menos de 48 horas.

\subsection{Resultados de las medidas registradas}

El gráfico de la figura 24 recoge las deformaciones medias circunferenciales de las dos secciones instrumentadas, sección a $1 / 4$ y sección en clave, en un periodo comprendido entre unas horas antes de empezar el hormigonado y dos días después de finalizar el mismo.

Como puede observarse, las deformaciones circunferenciales en la sección $1 / 4$ y en la clave son similares, con un pequeño desfase debido a los tiempos de hormigonado del arco. Esta similitud en las deformaciones medidas indica que el hormigón ha sido homogéneo con la dosis de aditivo expansivo utilizado y corrobora los distintos ensayos realizados en laboratorio con este tipo y dosis de aditivo.

Los gráficos de la figura 25 recogen la deformación longitudinal medida en cada uno de los puntos instrumentados de las secciones situadas a $1 / 4$ y en la clave. 


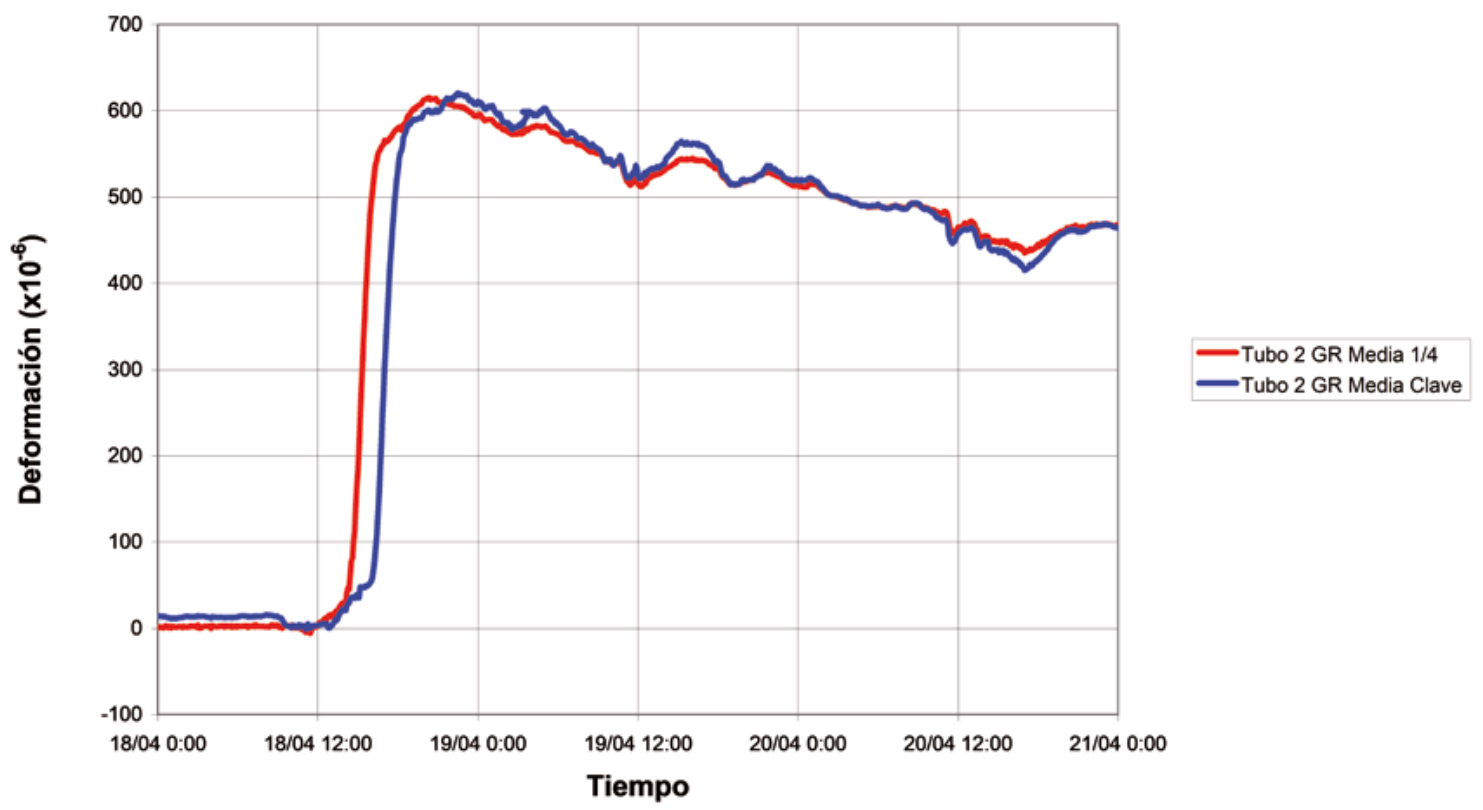

Figura 24. Deformación media circunferencial en sección clave y 1/4.

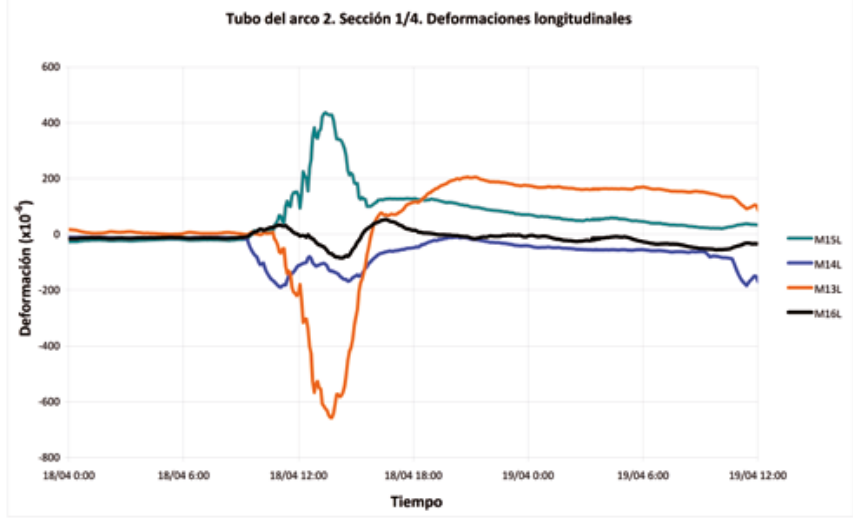

Sección $1 / 4$

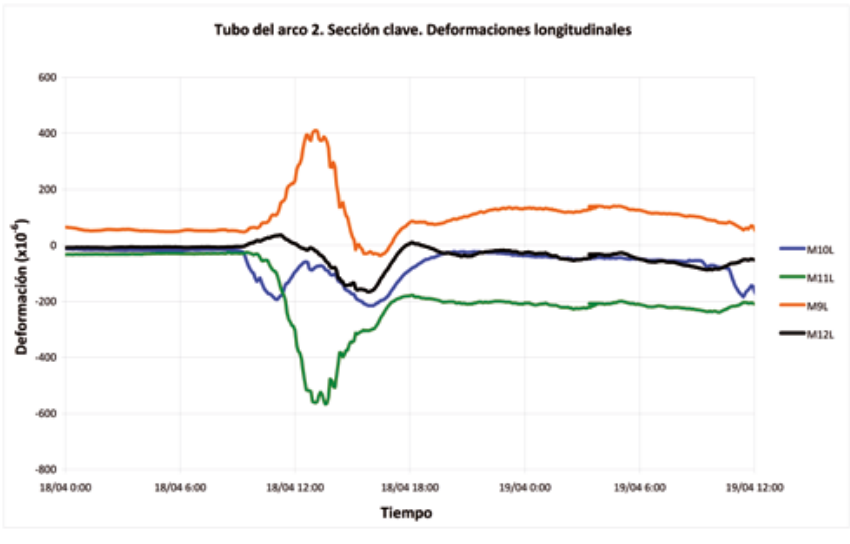

Sección clave

Figura 25. Deformación longitudinal en sección 1/4 y sección clave.

Como se observa en el gráfico, las deformaciones registradas en las bandas superior e inferior son de distinto signo, debido a la variación de los esfuerzos de flexión que se producen en la sección cuando se va hormigonando el arco.

El gráfico de la figura 26 muestra las deformaciones medias longitudinales de las dos secciones instrumentadas.

En el gráfico se observa también un desfase debido a los tiempos de hormigonado del arco. Las deformaciones longitudinales muestran el mismo ritmo de variación a lo largo del tiempo, estando influidas durante el hormigonado por los distintos esfuerzos de flexión que se producen en cada una de las secciones instrumentadas.

La deformación circunferencial media estabilizada obtenida en el tubo ha sido de $4.66 \cdot 10^{-4}$. Esta deformación ha sido menor que la registrada en los ensayos, algo esperable debido, entre otras circunstancias, a las condiciones favorables con la que se realizan los ensayos de laboratorio y a unas condiciones medioambientales uniformes y constantes de temperatura y humedad. Por otra parte, las condiciones de producción y ejecución en obra son muy distintas a las que se dan en laboratorio.

Utilizando el modelo teórico expuesto en el apartado 2 se puede determinar aproximadamente la presión de confinamiento que corresponde a las deformaciones medidas y la tensión de deslizamiento. Para las deformaciones medidas en los ensayos se ha obtenido una tensión de deslizamiento media de $0.846 \mathrm{~N} / \mathrm{mm}^{2}$, que disminuye aproximadamente un $22 \%$, hasta $0.663 \mathrm{~N} / \mathrm{mm}^{2}$ debido a los efectos térmicos. Para la deformación estabilizada medida in situ esta tensión puede estimarse en $0.605 \mathrm{~N} / \mathrm{mm}^{2}$, que pudiera disminuir a $0.474 \mathrm{~N} / \mathrm{mm}^{2}$ si se aplica la reducción producida por el calentamiento diferencial obtenida en los ensayos.

Las tensiones rasantes de cálculo más desfavorables varian a lo largo del arco, siendo la máxima para el ELU de $0.290 \mathrm{~N} / \mathrm{mm}^{2}$, menor que la tensión resistente estimada. Se obtiene un coeficiente adicional frente a las de tensiones del orden de 1.63. 


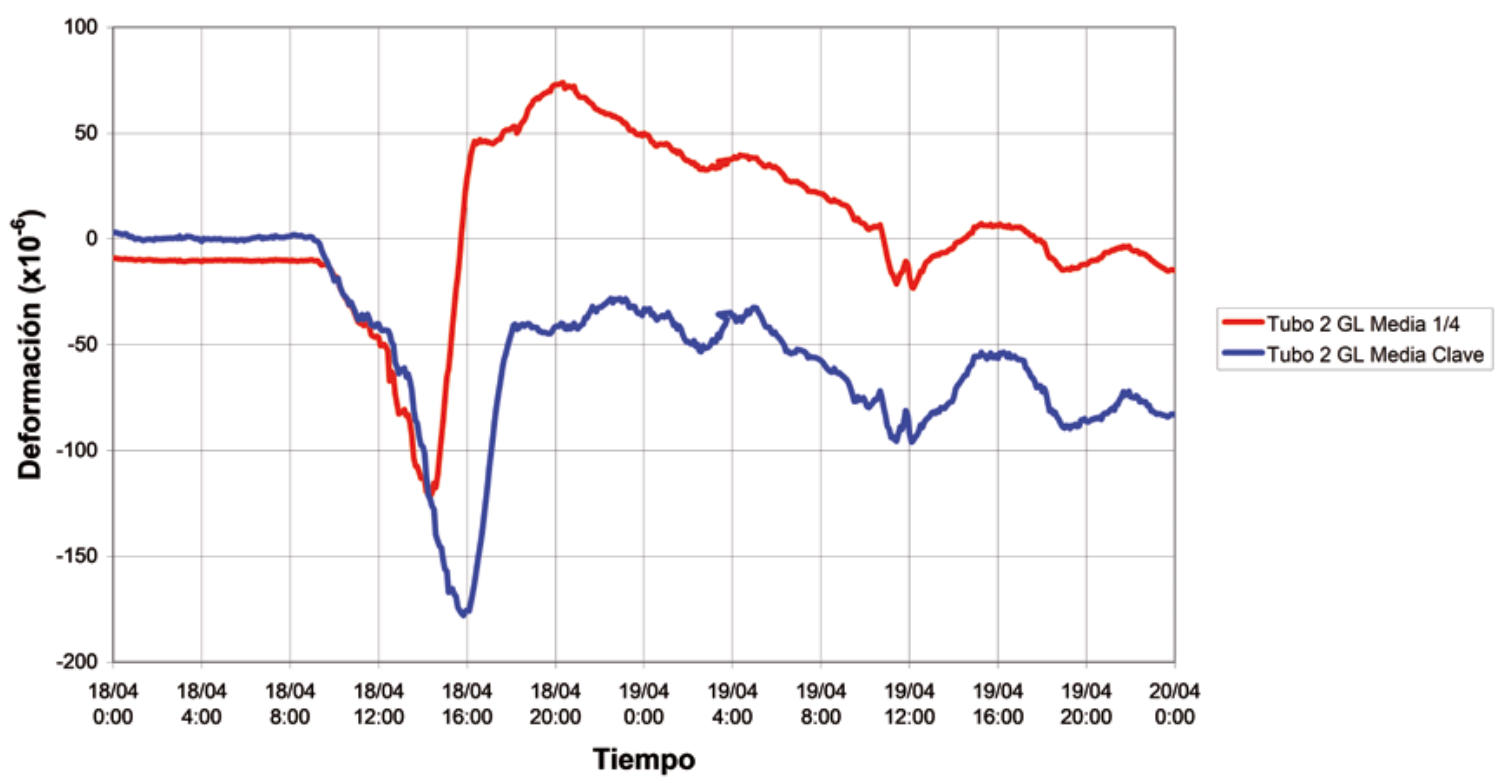

Figura 26. Tubo arco 2. Deformación media longitudinal en sección clave y 1/4.

Tubos de los arcos 1 y 2. Deformación circunferencial media

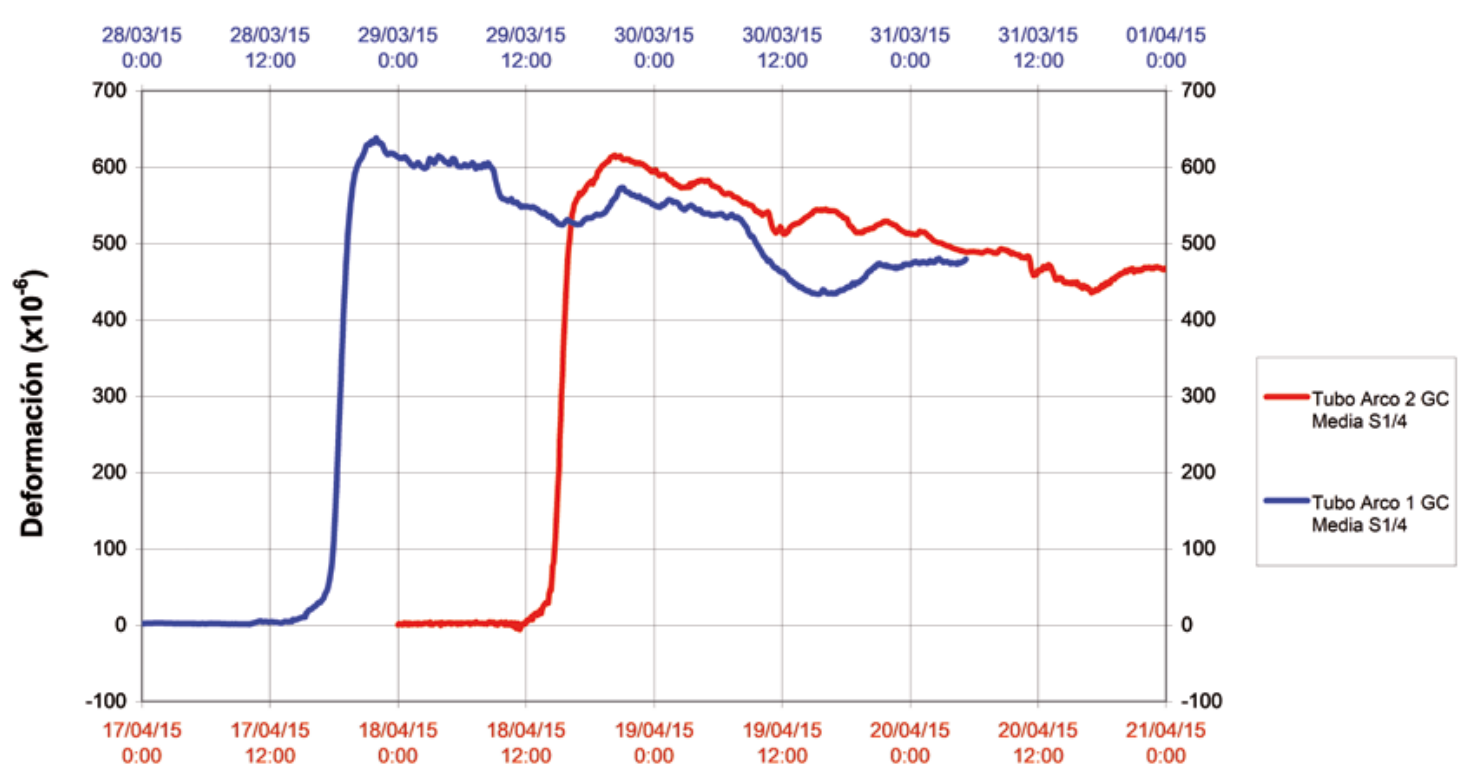

Figura 27. Deformación circunferencial media registrada en la sección 1/4 del tubo del arco 1 y en la sección a 1/4 del tubo del arco 2.

\subsection{Comparación de las deformaciones en los arcos}

La figura 27 recoge las deformaciones circunferenciales medias, en los primeros días después del hormigonado, de la sección instrumentada a $1 / 4$ del tubo del arco 1 , primer arco hormigonado, y en la sección instrumentada a 1/4 del tubo del arco 2 , hormigonado varios días después.

Como se observa en el gráfico de esta figura, las expansiones registradas en las dos secciones señaladas son muy si- milares, del mismo orden de magnitud, como corresponde a hormigones de las mismas características. Las deformaciones producidas son prácticamente iguales y la evolución en el tiempo es similar. La estabilización de las deformaciones se produce varios días después del hormigonado.

Como resumen, en el tubo del arco 1 , se ha registrado una deformación circunferencial media máxima del orden de $631 \cdot 10^{-6}$. Esta deformación tiende a estabilizarse a un valor del orden de $466 \cdot 10^{-6}$ pasados más de diez días del hormigonado. 
La deformación cíclica debida a los cambios térmicos que se produce a lo largo del día fue del orden de $\pm 40 \cdot 10^{-6}$. Las deformaciones longitudinales medidas reflejan los esfuerzos de compresión y flexión que se producen durante el hormigonado. Una vez estabilizada, la deformación longitudinal media registrada fue del orden de $-70 \cdot 10^{-6}$. La temperatura máxima alcanzada en el exterior del tubo del arco 1 debido al calor de hidratación ha sido de $60^{\circ} \mathrm{C}$.

En el tubo del arco 2 estos valores han sido respectivamente: $617 \cdot 10^{-6}$ como deformación circunferencial media máxima; $438 \cdot 10^{-6}$ como deformación circunferencial media estabilizada; $\pm 42 \cdot 10^{-6}$ como deformación cíclica debida a los cambios térmicos diarios y $-87 \cdot 10^{-6}$ como deformación longitudinal media, así como una temperatura máxima alcanzada en el exterior del tubo ha sido de $61^{\circ} \mathrm{C}$.

La deformación media estabilizada obtenida en los ensayos de laboratorio en los 9 tubos ensayados fue de $6,55 \cdot 10^{-4}$, y la tensión media de deslizamiento a temperatura ambiente de $0,846 \mathrm{~N} / \mathrm{mm}^{2}$, tensión que por el efecto del calentamiento diferencial de temperatura queda reducida a $0,663 \mathrm{~N} / \mathrm{mm}^{2}$ (del orden del $22 \%$ menor).

\section{5.}

\section{CONCLUSIONES}

Los sistemas mixtos CFT ("concrete filled tubes") rellenos con hormigón expansivo presentan ventajas desde el punto de vista estructural respecto al sistema tradicional de relleno con un hormigón convencional. Se produce una mejora de la capacidad resistente frente a las tensiones rasantes que se desarrollan en la interfase de ambos materiales. Estas tensiones son función de la deformación de expansión provocada en el hormigón, de la rigidez del tubo y del rozamiento entre ambos materiales.

El IETcc y Ferrovial Construcción, S.A. han colaborado en una investigación sobre hormigones autocompactantes expansivos para su aplicación en el relleno de los tubos metálicos de los arcos del viaducto del Barranco de Erques (Tenerife), desarrollando un programa experimental que ha permitido evaluar los parámetros fundamentales que rigen el comportamiento de este tipo de sistema mixto. Este programa ha consistido en obtener unas dosificaciones de hormigón autocompactante que generasen una expansión cercana a la deseada; realizar ensayos a deslizamiento con tubos de relación diámetro/espesor similar a los proyectados para los arcos del viaducto; así como llevar a cabo una instrumentación in situ de los arcos para evaluar las deformaciones y temperaturas que se producen en los tubos durante el hormigonado, fraguado y endurecimiento del hormigón.

Los resultados obtenidos de los ensayos de expansión libre y confinada sirvieron para ajustar la dosis del aditivo expansivo que permitiera conseguir la deformación necesaria prevista en el proyecto del puente arco. Los ensayos de autocompactabilidad sirvieron para asegurar un buen llenado de los tubos.

Los ensayos de deslizamiento, tanto a temperatura ambiente como con calentamiento exterior uniforme y diferencial, permitieron evaluar la influencia de la temperatura en la resistencia a deslizamiento del núcleo de hormigón. La tensión media de deslizamiento del núcleo disminuye un $15 \%$ para el caso de calentamiento uniforme y un $22 \%$ para el caso de calentamiento diferencial, respecto a la tensión media obtenida en los ensayos a temperatura ambiente de laboratorio. Aun así, la tensión remanente garantiza una buena conexión entre el hormigón y el acero.

Respecto a la instrumentación in situ se dispusieron galgas en la dirección circunferencial y longitudinal, así como termopares en cuatro puntos de cada sección (sección 1/4 y sección clave en cada uno de los tubos), lo que se completó con la instalación de fibra óptica para controlar una sección de uno de los tubos de un arco. Las deformaciones registradas en estas secciones resultaron del mismo orden de magnitud, como corresponde a un hormigón de las mismas características, diseñado para el hormigonado de los arcos. Los resultados obtenidos son acordes con las investigaciones previas realizadas en laboratorio sobre tubos de acero de similar relación diámetro/ espesor, obteniéndose cargas de deslizamiento que aseguran una capacidad suficiente a esfuerzo rasante para resistir las tensiones de cálculo deducidas del análisis estructural.

Este viaducto es un hito tecnológico de la ingeniería estructural española, siendo el primer puente en utilizar la tecnología del hormigón expansivo como relleno de los tubos de acero que constituyen los arcos portantes del tablero, prescindiendo del uso de conectores interiores.

\section{Agradecimientos}

Este trabajo se ha desarrollado dentro del Proyecto Intramural Especial, cuyo título es: "Análisis experimental y evaluación de sistemas estructurales" (ref.: 201760E066). Se agradece a Ferrovial Construcción, S.A. su colaboración con el IETcc para la realización de los trabajos, así como la participación del personal de los laboratorios y naves de ensayos estructurales del IETcc.

\section{Referencias}

[1] Moon, J., Lehman, D. E., Roeder, C. W., \& Lee, H. E. (2013) Strength of circular concrete-filled tubes with and without internal reinforcement under combined loading. Journal of Structural Engineering, 139 (12). https://ascelibrary.org/doi/pdf/10.1061/\%28ASCE\%29ST.1943-541X.0000788.

[2] Shanmugam, N. E., \& Lakshmi, B. (2001). State of the art report on steel-concrete composite columns. Journal of constructional steel research, 57(10), 1041-1080. https://www.sciencedirect.com/science/article/pii/ S0143974X01000219.

[3] Schneider, S. P. (1998). Axially loaded concrete-filled steel tubes. Journal of structural Engineering, 124(10), 1125-1138. https://ascelibrary.org/doi/ pdf/10.1061/(ASCE)0733-9445(1998)124\%3A10(1125).

[4] O'Shea, M. D., \& Bridge, R. Q. (2000). Design of Circular Thin-walled concrete filled steel tubes. Journal of Structural Engineering, 126(11), 1295-1303. https://ascelibrary.org/doi/pdf/10.1061/(ASCE)0733-9445 (2000)126\%3A11(1295).

[5] Hatzigeorgiou, G. D. (2008). Numerical model for the behavior and capacity of circular CFT columns, Part I: Theory. Engineering Structures, 30(6), 1573-1578. https://www.sciencedirect.com/science/article/pii/ S0141029607004063.

[6] Hatzigeorgiou, G. D. (2008). Numerical model for the behavior and capacity of circular CFT columns, Part II: Verification and extension. Engineering Structures, 30(6), 1579-1589. https://www.sciencedirect.com/ science/article/pii/S0141029607004117.

[7] de Oliveira, W. L. A., De Nardin, S., de Cresce El, A. L. H., \& El Debs, M. K. (2009). Influence of concrete strength and length/diameter on the axial capacity of CFT columns. Journal of Constructional Steel Research, 
65(12), 2103-2110. https://www.sciencedirect.com/science/article/pii/ S0143974X09001588.

[8] Chacón, R., Mirambell, E., \& Real, E. (2012). Capacidad Resistente de Pilas Metálicas Tubulares Circulares Rellenas de Hormigón (CFT) en Puentes Integrales. Informes de la Construcción, 64(527), 355-370. http:// informesdelaconstruccion.revistas.csic.es/index.php/informesdelaconstruccion/article/view/2281/2501.

[9] Susantha, K. A. S., Ge, H., \& Usami, T. (2001) Uniaxial stress-strain relationship of concrete confined by various shaped steel tubes. Engineering Structures, 23(10), 1331-1347. https://www.sciencedirect.com/science/ article/pii/S0141029601000207.

[10] Comité Europeo de Normalización, CEN (2013). UNE-EN 1994-1-1, Eurocódigo 4: Proyecto de Estructuras Mixtas de Acero y Hormigón. Parte 1-1: Reglas generales y Reglas para edificación.

[11] Comité Europeo de Normalización, CEN (2013). UNE-EN 1993-1-3, Eurocódigo 3: Proyecto de Estructuras de Acero. Parte 1-3: Reglas generales y Reglas adicionales para perfiles y chapas de paredes delgadas conformadas en frío.

[12] Comité Europeo de Normalización, CEN (2013). UNE-EN 1992-1-1, Eurocódigo 2: Proyecto de Estructuras de Hormigón. Parte 1-1: Parte 1-1: Reglas generales y Reglas para edificación.
[13] Comisión Permanente del Hormigón (2008). EHE-08, Instrucción de Hormigón Estructural. Ministerio de Fomento, Madrid.

[14] American Institute for Steel Construction, AISC (2010). Specification for structural steel buildings. Chicago.

[15] de Miguel, C., Pedro, G. C., Luis, J., \& Revuelta Crespo, D. (2017). Guía para el empleo de hormigones expansivos. Guía para el empleo de hormigones expansivos. Monografias del IETcc, $\mathrm{n}^{\circ} 425$, p. 1-63.

[16] Model Code for Concrete Structures 2010, fib CEB-FIP (2013).

[17] American Society for Testing and Materials, ASTM International (2008). ASTM C157/C157M-08, Standard Test Method for Length Change of Hardened Hydraulic-Cement Mortar and Concrete. (Última revisión: ASTM C157/C157M-17 de 2017)

[18] American Society for Testing and Materials, ASTM International (2009). ASTM C878/C878M -09, Standard Test Method for Restrained Expansion of Shrinkage-Compensating Concrete. (Última revisión: ASTM C878/C878M-14a de 2016).

[19] Comité Europeo de Normalización, CEN (2011). UNE-EN 12350-8, Ensayos de hormigón fresco. Parte 8: Hormigón autocompactante. Ensayo del escurrimiento. (Última revisión: 2020). 